\title{
FUNDAMENTAL GROUPS OF TANGENT CONIC-LINE ARRANGEMENTS WITH SINGULARITIES UP TO ORDER 6
}

\author{
MEIRAV AMRAM ${ }^{1}$, DAVID GARBER ${ }^{2}$ AND MINA TEICHER \\ Abstract. We list all the possible fundamental groups of the \\ complements of real conic-line arrangements with two conics which \\ are tangent to each other at two points, with up to two additional \\ lines. \\ For the computations we use the topological local braid mon- \\ odromies and the techniques of Moishezon-Teicher and van-Kampen. \\ We also include some conjectures concerning the connection be- \\ tween the presentation of the fundamental group of the comple- \\ ments and the geometry of an interesting family of conic line ar- \\ rangements.
}

\section{INTRODUCTION}

In this paper we compute and list the fundamental groups of complements of all real conic-line arrangements in $\mathbb{C P}^{2}$ with two conics, which are tangent to each other at two points, with up to two additional lines in any position.

Algorithmically, this paper uses the local computations (local braid monodromies and their induced relations), the braid monodromy techniques of Moishezon-Teicher (see [4, [6], 7], 8], [9] and [10]), the Enriques - van Kampen Theorem (see [11) and some group calculations for studying the fundamental groups. See [1] for detailed exposition of these techniques.

These arrangements may appear in a branch curve of a generic projection to $\mathbb{C P}^{2}$ of a surface of general type (see for example [5]).

The main results of this paper are as follows (where $e$ is the unit element in the group and $\mathbb{F}_{2}$ is the free group with two generators):

\footnotetext{
${ }^{1}$ Partially supported by the Minerva Foundation of Germany, the DAAD fellowship (Germany), EAGER (EU Network, HPRN-CT-2009-00099) and the Golda Meir postdoctoral fellowship.

${ }^{2}$ Partially supported by Lady Davis and Golda Meir postdoctoral fellowships.

Date: August 9, 2021.
} 
Proposition 1.1. Let $S$ be a curve in $\mathbb{C P}^{2}$ composed of two tangent conics. Then:

$$
\pi_{1}\left(\mathbb{C P}^{2}-S, *\right) \cong\left\langle x_{1}, x_{2} \mid\left(x_{1} x_{2}\right)^{2}=\left(x_{2} x_{1}\right)^{2}=e\right\rangle
$$

Proposition 1.2. There are three possible fundamental groups for a conic-line arrangement which consists of two tangent conics and an additional line:

(1) $\mathbb{Z} \oplus\left\langle x_{1}, x_{2} \mid\left(x_{1} x_{2}\right)^{2}=\left(x_{2} x_{1}\right)^{2}=e\right\rangle$.

(2) $\left\langle x_{1}, x_{2} \mid\left(x_{1} x_{2}\right)^{2}=\left(x_{2} x_{1}\right)^{2}\right\rangle$.

(3) $\mathbb{F}_{2}$

Proposition 1.3. There are five possible fundamental groups for a conic-line arrangement which consists of two tangent conics and two additional lines:

(1)

$$
\left\langle\begin{array}{c|c}
x_{1}, x_{2}, x_{3} & \left(x_{2} x_{3}\right)^{2}=\left(x_{3} x_{2}\right)^{2},\left(x_{1} x_{3}\right)^{2}=\left(x_{3} x_{1}\right)^{2}, \\
{\left[x_{1}, x_{2}\right]=\left[x_{2}, x_{3} x_{1} x_{3}^{-1}\right]=e}
\end{array}\right\rangle
$$

(2) $\left\langle x_{1}, x_{2}, x_{3} \mid\left(x_{3} x_{2} x_{1}\right)^{2}=\left(x_{2} x_{1} x_{3}\right)^{2}=\left(x_{1} x_{3} x_{2}\right)^{2}\right\rangle$

(3) $\mathbb{Z} \oplus\left\langle x_{1}, x_{2} \mid\left(x_{1} x_{2}\right)^{2}=\left(x_{2} x_{1}\right)^{2}\right\rangle$

(4) $\mathbb{Z} \oplus \mathbb{F}_{2}$

(5) $\mathbb{Z}^{2} \oplus\left\langle x_{1}, x_{2} \mid\left(x_{1} x_{2}\right)^{2}=\left(x_{2} x_{1}\right)^{2}=e\right\rangle$

The proof of Proposition 1.1 is presented completely in Section 3 . Otherwise, we skip all the braid monodromy computations, and give only the presentation of the fundamental group obtained by the van Kampen Theorem. These computations and the proofs of Propositions 1.2 and 1.3 appear in Sections 4 and 5 , respectively.

A group is called big if it contains a subgroup which is free (generated by two or more generators). By the above results, we have the following corollary:

Corollary 1.4. All the possibilities for fundamental groups of conicline arrangements consist of two tangent conics and up to two additional lines are big.

The proof of this corollary appears in Section 6.

We rule out some possibilities for conic-line arrangements by the following remark.

Remark 1.5. The only possibility for a line to be tangent to both conics is that this line will pass through one of the tangency points of the two conics (fourth case in Section [4.2). The reason is the following. If a line was tangented to both conics, it would be a common point of the 
two duals to the two conics. Now, since the dual curve of two tangented conics is two tangented conics too, there are only two common points to the two dual conics. These two common points correspond to the two tangency points between the original two conics. Hence, a different line which tangents to both conics is impossible.

The paper is organized as follows.

Section 2 presents the local computations related to the singular points appearing in the conic-line arrangements which we deal with.

Section 3 deals with the proof of Proposition 1.1. In Section 4 we compute the different cases of conic-line arrangements which consist of two tangent conics and one additional line. Section 5 deals with the different cases of conic-line arrangements which consist of two tangent conics and two additional lines. In Section 6 we show the simple proof of Corollary 1.4.

Section 7 deals with some conjectures concerning the connection between the presentation of the fundamental group of the complements and the geometry of an interesting family of conic-line arrangements.

In the appendix, we list all the possibilities for two tangent conics and two additional lines with the corresponding fundamental groups of their complements.

\section{LOCAL COMPUTATIONS}

In this section, we present the local computations related to the singular points appeared in the conic-line arrangements which we deal with.

2.1. A singular point with two components. In this section, we compute the local braid monodromy of a tangency point of two conics.

The tangency point between two conics can be presented locally by the equation: $\left(y+x^{2}\right)\left(y-x^{2}\right)=0$ (see Figure 1).

The singular point is $(0,0)$, and the points of the curve in the fiber over $x=1$ are $y=1$ and $y=-1$. Now, let us take a loop in $x$-axis based at $x=1$ and circumscribing $0: \alpha(t)=e^{2 \pi i t}$, where $0 \leq t \leq$ 1. Lifting it to the curve, we get two paths on the curve: $\alpha_{1}(t)=$ $\left(e^{2 \pi i t}, e^{4 \pi i t}\right)$ and $\alpha_{2}(t)=\left(e^{2 \pi i t},-e^{4 \pi i t}\right)$ where $0 \leq t \leq 1$. Projecting the paths to the $y$-axis, we get $y_{1}(t)=e^{4 \pi i t}$ and $y_{2}(t)=-e^{4 \pi i t}$ where $0 \leq t \leq 1$. By substituting $t=1$, the local braid monodromy of this point is two counterclockwise full-twists. Hence, we have:

Theorem 2.1. The local braid monodromy of the tangency point of two conics is two counterclockwise full-twists (similar to the tangency point 


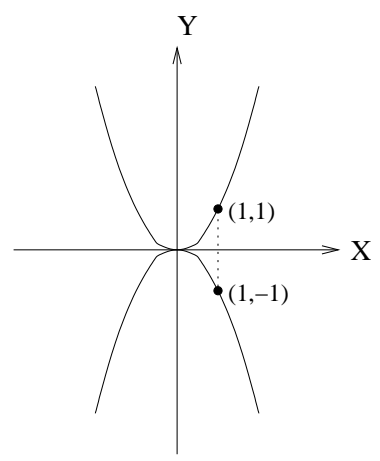

Figure 1. The singularity of $\left(y+x^{2}\right)\left(y-x^{2}\right)=0$ at $(0,0)$

of a conic and a line). Hence, we have the same induced relations:

$$
\left(x_{1} x_{2}\right)^{2}=\left(x_{2} x_{1}\right)^{2}
$$

where $\left\{x_{1}, x_{2}\right\}$ are the generators of the standard g-base.

Now, we compute the corresponding Lefschetz diffeomorphism:

Corollary 2.2. The Lefschetz diffeomorphism of a tangency point between two tangent conics is a counterclockwise full-twist (similar to the usual tangency point).

The reason for this corollary is since the Lefschetz diffeomorphism is obtained by going on the loop mentioned above from $t=\frac{1}{2}$ to $t=1$. Along this interval, the two points in the fiber make one counterclockwise full-twist, and hence the Lefschetz diffeomorphism of this singular point is a full-twist.

2.2. Singular points with three components. In this section, we compute the local braid monodromy of four types of singular points consist of three components (where at most two of them are conics which are tangent). Then, we compute the relations induced from these singular points by the classical van Kampen Theorem (see [1]]).

2.2.1. First type. The local equation of the singularity of the first type is $(2 x+y)\left(y+x^{2}\right)\left(y-x^{2}\right)=0$ (see Figure 2).

For computing the braid monodromy, we take a loop around $x=0$ and look at what happens to the points of the curve $(2 x+y)\left(y+x^{2}\right)(y-$ $\left.x^{2}\right)=0$ in the fibers above this loop.

Let $x=e^{2 \pi i t}$ where $0 \leq t \leq 1$. For $t=0$ we have that $x=1$ and the points of the curve in the fiber over $x=1$ are $y=1,-1$ and $y=-2$. For $t=\frac{1}{2}$, we have $x=-1$, and the points of the curve in the fiber over $x=-1$ are $y=-1,1$ and $y=2$. The point $y=1$ in the fiber 


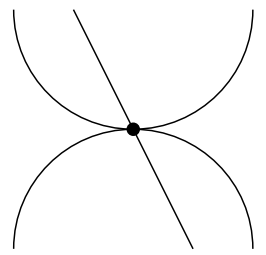

FiguRE 2. The singularity of $(2 x+y)\left(y+x^{2}\right)\left(y-x^{2}\right)=0$ at $(0,0)$

$x=-1$ corresponds to the point $y=1$ in the fiber $x=1$, and similarly the point $y=-1$ in the fiber $x=-1$ corresponds to the point $y=-1$ in the fiber $x=1$. The point $y=2$ in the fiber $x=-1$ corresponds to the point $y=-2$ in the fiber $x=1$. Hence, we get that from $t=0$ to $t=\frac{1}{2}$, the points $y=1$ and $y=-1$ do a counterclockwise fulltwist and the point $y=-2$ does a counterclockwise half-twist around the points $y=1,-1$. If we continue to $t=1$, the points $y=1$ and $y=-1$ do two counterclockwise full-twists and the point $y=-2$ does a counterclockwise full-twist around the points $y=1,-1$ together.

Now, we want to compute the induced relations from the braid monodromy of the singular point. According to van Kampen's Theorem, one should compute the g-base obtained by applying the action induced by the local braid monodromy of the singular point on the standard g-base. This is shown in Figure 3. in the first step, we perform two counterclockwise full-twists of points 2 and 3 which represent $y=-1$ and $y=1$ respectively. In the second step, we perform a counterclockwise full-twist of point 1 (which represents the point $y=-2$ ) around the points 2 and 3 .

Now, by van Kampen's Theorem, we get the following induced relations from the new g-base (where $\left\{x_{1}, x_{2}, x_{3}\right\}$ are the generators of the standard g-base):

(1) $x_{1}=x_{3} x_{2} x_{1} x_{2}^{-1} x_{3}^{-1}$

(2) $x_{2}=x_{3} x_{2} x_{1} x_{3} x_{2} x_{3}^{-1} x_{1}^{-1} x_{2}^{-1} x_{3}^{-1}$

(3) $x_{3}=x_{3} x_{2} x_{1} x_{3} x_{2} x_{3} x_{2}^{-1} x_{3}^{-1} x_{1}^{-1} x_{2}^{-1} x_{3}^{-1}$

From Relation (1), we get that $x_{1} x_{3} x_{2}=x_{3} x_{2} x_{1}$. Relation (2) becomes $x_{3} x_{2} x_{1} x_{3} x_{2}=x_{2} x_{3} x_{2} x_{1} x_{3}$.

Now, we show that Relation (3) is not needed. Simplifying Relation (3) yields $x_{2} x_{1} x_{3} x_{2} x_{3}=x_{3} x_{2} x_{1} x_{3} x_{2}$. By Relation (1), this relation is equal to Relation (2), and hence Relation (3) is redundant.

To summarize, we proved the following:

Theorem 2.3. The local braid monodromy of the singularity presented locally by the equation $(2 x+y)\left(y+x^{2}\right)\left(y-x^{2}\right)=0$ is: two points 

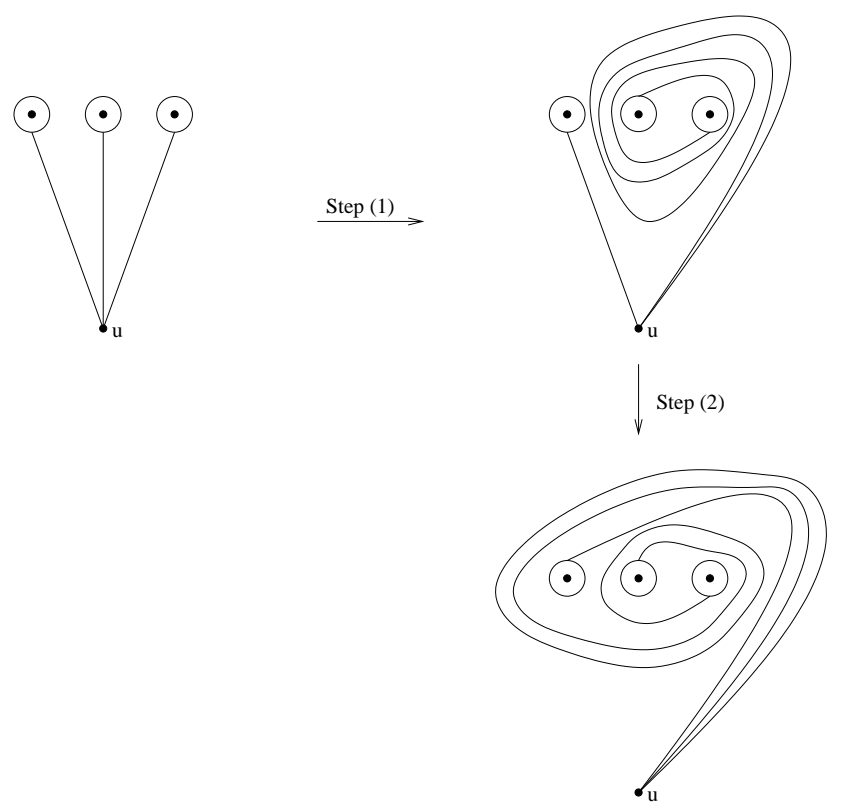

FiguRE 3. The g-base obtained from the standard gbase by the action of the local braid monodromy

(corresponding to $y=1$ and $y=-1$ ) do two counterclockwise full-twists and the third point (corresponds to $y=-2$ ) does a counterclockwise full-twist around them.

The induced relations of this point are:

$$
x_{1} x_{3} x_{2}=x_{3} x_{2} x_{1} \quad ; \quad x_{3} x_{2} x_{1} x_{3} x_{2}=x_{2} x_{3} x_{2} x_{1} x_{3}
$$

where $\left\{x_{1}, x_{2}, x_{3}\right\}$ are the generators of the standard $g$-base.

Since the Lefschetz diffeomorphism of the singular point is obtained by computing the action only on half of the unit circle (from $t=\frac{1}{2}$ to $t=1$ ), we have the following corollary:

Corollary 2.4. The Lefschetz diffeomorphism of the singular point presented locally by $(2 x+y)\left(y+x^{2}\right)\left(y-x^{2}\right)=0$ is a counterclockwise full-twist of the points corresponding to $y=1$ and $y=-1$, and $a$ half-twist of the point corresponding to $y=2$ (which becomes $y=-2$ ) around them.

Remark 2.5. One can easily see that the singular point presented locally by the equation $y(2 x+y)\left(y+x^{2}\right)=0$ (see Figure 4) has the same braid monodromy and the same induced relations as the singular point which we have dealt with in this section, since these two singular points are locally the same (from the topological point of view). 


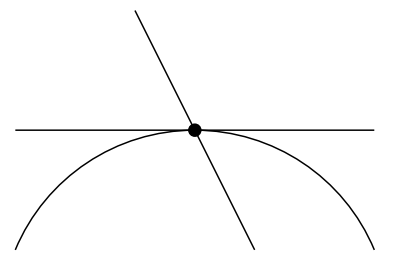

Figure 4. The singularity of $y(2 x+y)\left(y+x^{2}\right)=0$ at $(0,0)$

2.2.2. Second type. The local equation of the singularity of the second type is $(2 x-y)\left(y+x^{2}\right)\left(y-x^{2}\right)=0$ (see Figure 5).

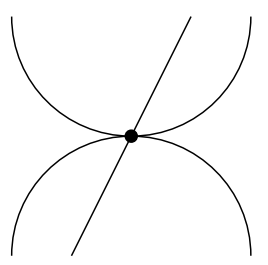

FiguRE 5. The singularity of $(2 x-y)\left(y+x^{2}\right)\left(y-x^{2}\right)=0$ at $(0,0)$

Applying almost the same computations we have performed for the first type, we get the following result:

Theorem 2.6. The local braid monodromy of the singularity presented locally by the equation $(2 x-y)\left(y+x^{2}\right)\left(y-x^{2}\right)=0$ is: two points (corresponding to $y=1$ and $y=-1$ ) do two counterclockwise full-twists and the third point (corresponding to $y=2$ ) do a counterclockwise fulltwist around them.

The corresponding induced relations are:

$$
x_{3} x_{2} x_{1}=x_{2} x_{1} x_{3} \quad ; \quad x_{3} x_{2} x_{1} x_{2} x_{1}=x_{1} x_{3} x_{2} x_{1} x_{2}
$$

where $\left\{x_{1}, x_{2}, x_{3}\right\}$ are the generators of the standard $g$-base.

The Lefschetz diffeomorphism of the singular point is a counterclockwise full-twist of the points corresponding to $y=1$ and $y=-1$, and a half-twist of the point corresponding to $y=-2$ (which becomes $y=2$ ) around them.

Remark 2.7. As in the previous section, one can easily see that the singular point presented locally by the equation $y(2 x-y)\left(y+x^{2}\right)=0$ (see Figure 6) has the same braid monodromy and the same induced relations as the singular point which we have dealt with in this section, since these two singular points are locally the same. 


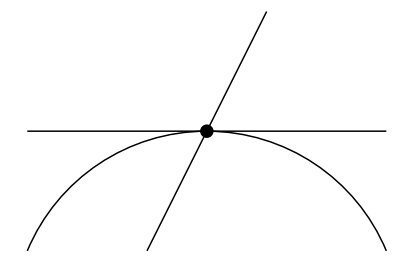

FIGURE 6 . The singularity of $y(2 x-y)\left(y+x^{2}\right)=0$ at $(0,0)$

2.2.3. Third type. The local equation of the singularity of the third type is $y\left(y^{2}+x\right)\left(y^{2}-x\right)=0$ (see Figure 17). One should notice that there is a major difference between this type of singularity and the two previous types: In this singularity there are two "hidden" branch points. That is, at any fiber one has three real points and two complex points (i.e. complex level 2), and in each side of the singularity, the complex points belong to a different conic (by the singularity, two real points become complex and two complex points become real).

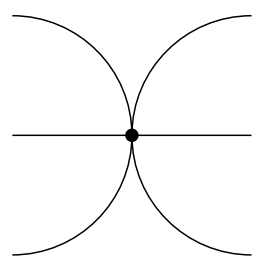

FiguRE 7 . The singularity of $y\left(y^{2}+x\right)\left(y^{2}-x\right)=0$ at $(0,0)$

For computing the braid monodromy, we take a loop around $x=0$ and look what happens to the points of the curve $y\left(y^{2}+x\right)\left(y^{2}-x\right)=0$ in the fibers over this loop.

Let $x=e^{2 \pi i t}$ where $0 \leq t \leq 1$. For $t=0$ we have that $x=1$ and the points of the curve in the fiber over $x=1$ are $y=-1,0,1, i$ and $y=-i$. For $t=\frac{1}{2}$, we have $x=-1$, and we get again that the points of the curve in the fiber over $x=-1$ are $y=-1,0,1, i$ and $y=-i$. By a careful checking, one can see that the point $y=1$ in the fiber $x=-1$ corresponds to the point $-i$ in the fiber $x=1$, and similarly all the points except for $y=0$ in the fiber $x=-1$ made a $90^{\circ}$ rotation counterclockwise around $y=0 \in \mathbb{C}$ from their corresponding points in the fiber $x=1$. The point $y=0$ remains fixed. Now, when we continue to $t=1$, the points of the fiber continue to move counterclockwise around $y=0$. When we reach $t=1$ and reach back the fiber $x=1$, the points in the fiber made a $180^{\circ}$ rotation counterclockwise around $y=0 \in \mathbb{C}$ from their corresponding points in the initial fiber $x=1$. Hence, we have: 
Theorem 2.8. The action of local braid monodromy of the point presented locally by the equation: $y\left(y^{2}+x\right)\left(y^{2}-x\right)=0$ is a $180^{\circ}$ rotation counterclockwise of the four points (corresponding to $y=1,-1, i,-i$ ) around the point corresponding to $y=0$, as shown schematically in Figure 8 .

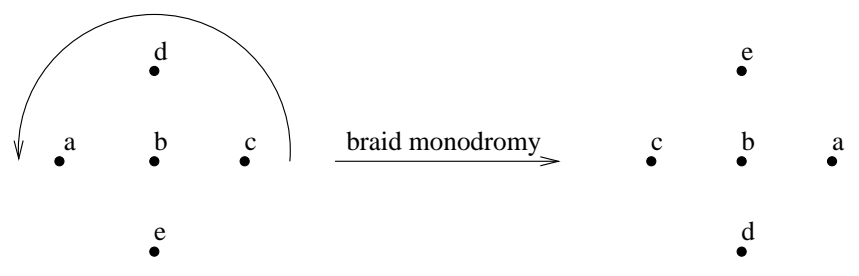

Figure 8 . The local braid monodromy of the singularity $y\left(y^{2}+x\right)\left(y^{2}-x\right)=0$

Now, we want to compute the induced relations of this singular point. By van Kampen's Theorem, one should compute the g-base obtained by applying the action induced by the local braid monodromy of the singular point on the standard g-base. Since we have two complex points in the fiber before the action of the braid monodromy and after it, we have to start by rotating the two rightmost points by $90^{\circ}$ counterclockwise, for representing the two complex points (see Step (1) in Figure 9). Then, we move the two complex points to be $i$ and $-i$ (see Step (2) there). Now, we apply the action of the local braid monodromy (Step (3)), and then we return the two new complex points to the right side, and return them to the real axis by rotating them by $90^{\circ}$ clockwise (Step (4)).

Remark 2.9. One should notice here that when we return the points back to the real axis in Step (4), we rotate the points clockwise. The reason is that we want to compute the pure action of the braid on the $g$-base, and the clockwise rotation cancels the counterclockwise rotation which we have performed in Step (1). In the global computations, we indeed rotate always counterclockwise, since we work there in a model (for simplifying the computations), and we can define the model as we want. We get the same results in the global computations even if we rotate clockwise the complex points into real points.

Now, by van Kampen's Theorem, we get the following induced relations from the new g-base (where $\left\{x_{1}, x_{2}, x_{3}, x_{4}, x_{5}\right\}$ are the generators of the standard g-base):

(1) $x_{1}=x_{4} x_{3} x_{4}^{-1}$

(2) $x_{2}=x_{4} x_{3} x_{2} x_{3}^{-1} x_{4}^{-1}$ 


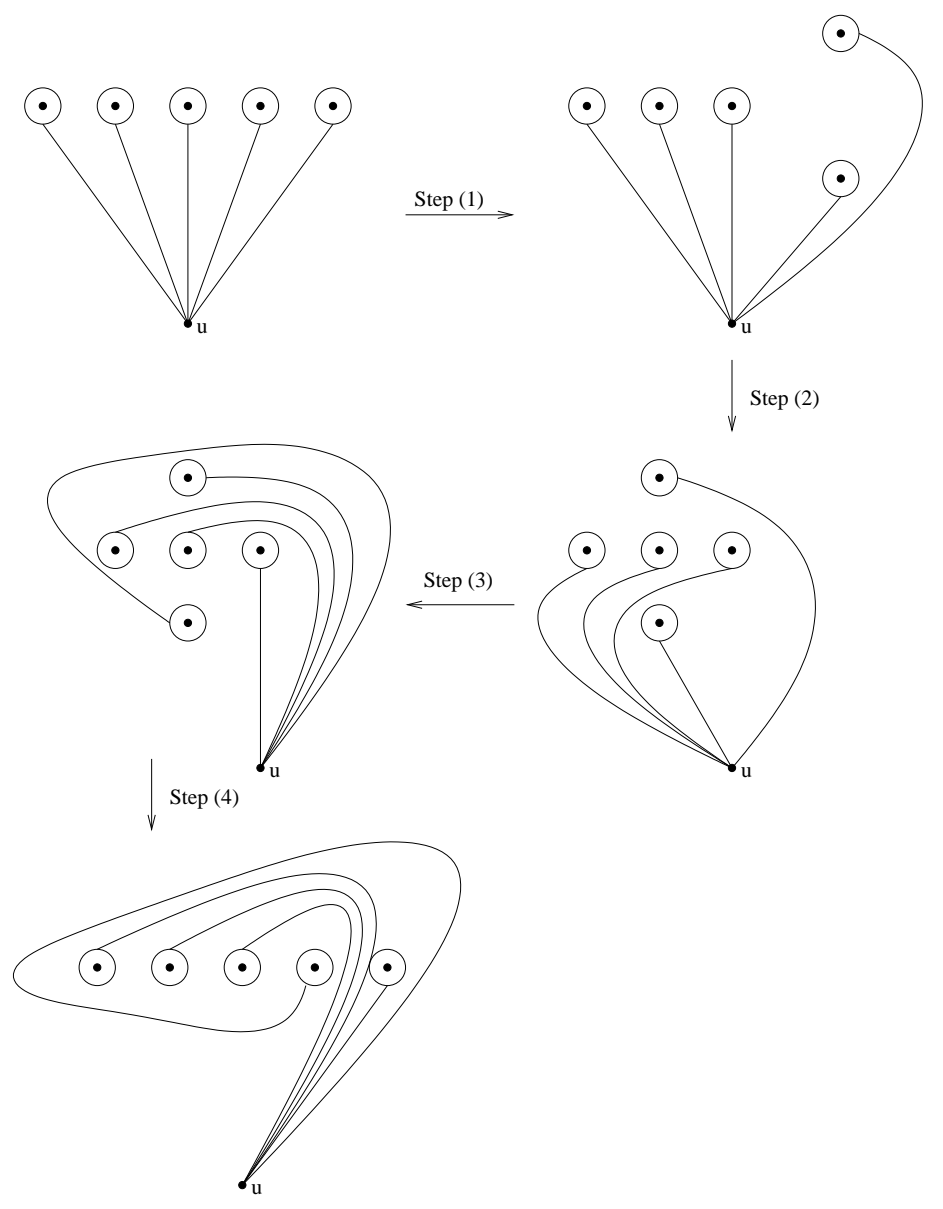

Figure 9. The g-base obtained from the standard gbase by the action of the local braid monodromy

(3) $x_{3}=x_{4} x_{3} x_{2} x_{1} x_{2}^{-1} x_{3}^{-1} x_{4}^{-1}$

(4) $x_{4}=x_{5}$

(5) $x_{5}=x_{5} x_{4} x_{3} x_{2} x_{1} x_{4} x_{1}^{-1} x_{2}^{-1} x_{3}^{-1} x_{4}^{-1} x_{5}^{-1}$

Relation (2) is equivalent to $x_{4} x_{3} x_{2}=x_{2} x_{4} x_{3}$. Substituting $x_{1}$ by $x_{4} x_{3} x_{4}^{-1}$ in Relation (3) yields $x_{3}=x_{4} x_{3} x_{2} x_{4} x_{3} x_{4}^{-1} x_{2}^{-1} x_{3}^{-1} x_{4}^{-1}$, which is equivalent to $x_{4} x_{3} x_{2} x_{4} x_{3}=x_{3} x_{4} x_{3} x_{2} x_{4}$. By Relation (2), it can also be written as $x_{4} x_{3} x_{2} x_{4} x_{3}=x_{3} x_{2} x_{4} x_{3} x_{4}$.

We show that Relation (5) is redundant. First, we can cancel $x_{5}$, so we get: $x_{4} x_{3} x_{2} x_{1} x_{4}=x_{5} x_{4} x_{3} x_{2} x_{1}$. Since $x_{4}=x_{5}$, we can cancel another $x_{5}$, and by substituting $x_{1}$ by $x_{4} x_{3} x_{4}^{-1}$ and some simplifications we get $x_{3} x_{2} x_{4} x_{3} x_{4}=x_{4} x_{3} x_{2} x_{4} x_{3}$, which is equal to Relation (3). Hence, Relation (5) is redundant.

Therefore, we have the following corollary: 
Corollary 2.10. The induced relations for the singular point presented locally by the equation $y\left(y^{2}+x\right)\left(y^{2}-x\right)=0$ are:

(1) $x_{4} x_{3} x_{2}=x_{2} x_{4} x_{3}$

(2) $x_{3} x_{2} x_{4} x_{3} x_{4}=x_{4} x_{3} x_{2} x_{4} x_{3}$

(3) $x_{1}=x_{4} x_{3} x_{4}^{-1}$

(4) $x_{4}=x_{5}$

where $\left\{x_{1}, x_{2}, x_{3}, x_{4}, x_{5}\right\}$ are the generators of the standard $g$-base.

Now, we compute the corresponding Lefschetz diffeomorphism:

Corollary 2.11. The Lefschetz diffeomorphism of the singular point presented locally by $y\left(y^{2}+x\right)\left(y^{2}-x\right)=0$ is a $90^{\circ}$ rotation counterclockwise of the four points around the fixed point $y=0 \in \mathbb{C}$.

2.2.4. Fourth type. This type is slightly different from the previous three types. In the previous types, the line intersects the tangency point, but was not tangent to it. In this type, the additional line and the tangency point have a common tangent.

The local equation of the singularity of this type is $y\left(y+x^{2}\right)\left(y-x^{2}\right)=$ 0 (see Figure [10).

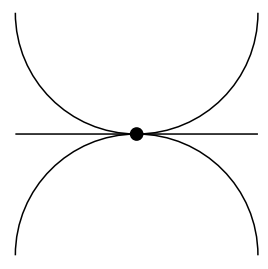

Figure 10. The singularity of $y\left(y+x^{2}\right)\left(y-x^{2}\right)=0$ at $(0,0)$

By similar arguments as in Theorem 2.1, we get

Theorem 2.12. The local braid monodromy of the singularity presented locally by the equation $y\left(y+x^{2}\right)\left(y-x^{2}\right)=0$ is: the three points do two counterclockwise generalized full-twists.

The induced relations of this point are:

$$
\left(x_{3} x_{2} x_{1}\right)^{2}=\left(x_{1} x_{3} x_{2}\right)^{2}=\left(x_{2} x_{1} x_{3}\right)^{2},
$$

where $\left\{x_{1}, x_{2}, x_{3}\right\}$ are the generators of the standard $g$-base.

The Lefschetz diffeomorphism is a full-twist of all the three points.

This result can be easily generalized to an arbitrary number of tangented components at the same point with a common tangent: 
Corollary 2.13. Let $P$ be a singular point which consists of $n$ smooth branches with a common tangent at $P$. Let $x_{i}$ be the generator which corresponds to the ith branch. Then, the local braid monodromy is two generalized full-twists of the whole segment $[1, n]$. Moreover, the relations induced by this singular point are:

$$
\left(x_{n} x_{n-1} \cdots x_{1}\right)^{2}=\left(x_{n-1} \cdots x_{1} x_{n}\right)^{2}=\cdots=\left(x_{1} x_{n} \cdots x_{2}\right)^{2} .
$$

Notice that if the tangented components have a higher order of tangency (i.e. the higher derivatives of the components are also equal up to the $k$ th derivative, for a given $k$ ), then the braid monodromy will consist of a higher power of a full-twist, and a higher exponent will appear in the relations.

2.3. Singular points with four components. In this section, we compute the local braid monodromy of five cases of singular points consist of four components, where at most two of them are conics. Then, we compute the relations induced from these singular points by the classical van Kampen Theorem (see [11]).

A singular point consists of four components in a conic-line arrangements with two tangent conics and up to two additional lines can be of the following two types: take the tangency point of the two conics, and add two lines in the following two ways: one way is to add one line which will be tangent to both conics, and the second line will intersect both conics (and the line) at the singular point. The second way will be to add two intersecting lines at the tangency point of the two conics.

2.3.1. Two tangent conics with a tangent line and an intersecting line. As before, there are three possibilities for the intersecting line: it can be locally presented as the line $y=2 x, y=-2 x$ or $x=0$. In spite of the fact that one can move from the first type to the second type by rotating the line, so locally the singularities are equivalent, from the global point of view these singularities induce different relations in the global fundamental group.

First type. The local equation of the singularity of the first type is $y(2 x+y)\left(y+x^{2}\right)\left(y-x^{2}\right)=0$ (see Figure 11).

By a similar argument as in Theorem 2.3, we have

Theorem 2.14. The local braid monodromy of the singularity presented locally by the equation $y(2 x+y)\left(y+x^{2}\right)\left(y-x^{2}\right)=0$ is: three points (correspond to $y=1,0$ and $y=-1$ ) do two counterclockwise generalized full-twists and the fourth point (corresponds to $y=-2$ ) do a counterclockwise full-twist around them. 


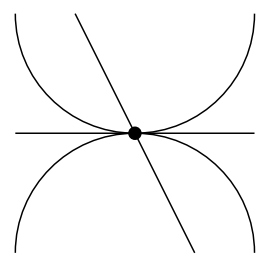

FiguRE 11. The singularity of $y(2 x+y)\left(y+x^{2}\right)\left(y-x^{2}\right)=$ 0 at $(0,0)$

The induced relations from this singular point are:

$x_{1} x_{4} x_{3} x_{2}=x_{4} x_{3} x_{2} x_{1} \quad ; \quad\left(x_{4} x_{3} x_{2}\right)^{2} x_{1}=x_{3} x_{2} x_{4} x_{3} x_{2} x_{1} x_{4}=x_{2} x_{4} x_{3} x_{2} x_{1} x_{4} x_{3}$, where $\left\{x_{1}, x_{2}, x_{3}, x_{4}\right\}$ are the generators of the standard $g$-base.

The Lefschetz diffeomorphism of the singular point is a counterclockwise generalized full-twist of the points correspond to $y=1,0$ and $y=-1$, and a half-twist of the point corresponds to $y=2$ (which becomes $y=-2$ ).

Remark 2.15. If we delete the generator $x_{1}$ which corresponds to the line that intersects the three tangent components, we get the following set of relations:

$$
\left(x_{4} x_{3} x_{2}\right)^{2}=\left(x_{3} x_{2} x_{4}\right)^{2}=\left(x_{2} x_{4} x_{3}\right)^{2},
$$

as expected (see Section 2.2.4). Similarly, if we delete the generator $x_{3}$ which corresponds to the tangent line, we get the following set of relations:

$$
x_{1} x_{4} x_{2}=x_{4} x_{2} x_{1} \quad ; \quad x_{4} x_{2} x_{4} x_{2} x_{1}=x_{2} x_{4} x_{2} x_{1} x_{4}
$$

again as expected (see Section 2.2.1).

Second type. The local equation of the singularity of the second type is $y(2 x-y)\left(y+x^{2}\right)\left(y-x^{2}\right)=0$ (see Figure 12).

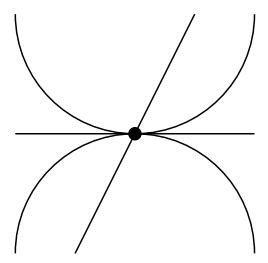

FigurE 12. The singularity of $y(2 x-y)\left(y+x^{2}\right)\left(y-x^{2}\right)=$ 0 at $(0,0)$

Applying almost the same computations we have performed for the previous type, we get: 
Theorem 2.16. The local braid monodromy of the singularity presented locally by the equation $y(2 x-y)\left(y+x^{2}\right)\left(y-x^{2}\right)=0$ is: the points $y=1,0,-1$ do two counterclockwise generalized full-twists, and the point $y=2$ does a counterclockwise full-twist around the points $y=1,0,-1$ together.

The corresponding induced relations are:

$x_{4} x_{3} x_{2} x_{1}=x_{3} x_{2} x_{1} x_{4} \quad ; \quad\left(x_{3} x_{2} x_{1}\right)^{2} x_{4}=x_{2} x_{1} x_{3} x_{2} x_{1} x_{4} x_{3}=x_{1} x_{3} x_{2} x_{1} x_{4} x_{3} x_{2}$, where $\left\{x_{1}, x_{2}, x_{3}, x_{4}\right\}$ are the generators of the standard $g$-base.

The Lefschetz diffeomorphism of the singular point presented locally by $y(2 x-y)\left(y+x^{2}\right)\left(y-x^{2}\right)=0$ is a counterclockwise generalized fulltwist of the points correspond to $y=1,0$ and $y=-1$, and a half-twist of the point corresponds to $y=-2$ (which becomes $y=2$ ).

Third type. The local equation of the singularity of the third type is $x y\left(y+x^{2}\right)\left(y-x^{2}\right)=0$ (see Figure 13).

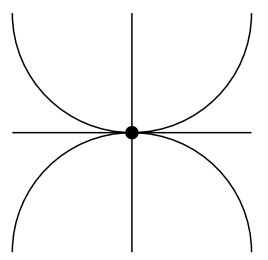

FIgURE 13 . The singularity of $x y\left(y+x^{2}\right)\left(y-x^{2}\right)=0$ at $(0,0)$

For computing the braid monodromy in this case, we use the following trick: the three components in the middle $x\left(y+x^{2}\right)\left(y-x^{2}\right)=0$ can be thought for a moment as a "thick" line which is perpendicular to the other line. After this observation, we have two intersecting lines, whose local braid monodromy is a counterclockwise full-twist (even though we have here a thick line $x=0$, which is vertical, one can rotate it a bit getting two "usual" intersecting lines, without changing the braid monodromy).

The local braid monodromy induced by the "thick" line itself (which consists of the curve $x\left(y+x^{2}\right)\left(y-x^{2}\right)=0$ ) has already been computed (Section 2.2.3): we got there that the local braid monodromy is a $180^{\circ}$ counterclockwise rotation of four points around one fixed point at the origin.

Hence, the action of the local braid monodromy of the whole singular point can be summarized as follows:

Theorem 2.17. The local braid monodromy of the singular point presented locally by the equation $x y\left(y+x^{2}\right)\left(y-x^{2}\right)=0$ is: first perform a $180^{\circ}$ counterclockwise rotation of the points correspond to $y=$ 
$1,-1, i,-i$ around $y=0$, and then the point corresponds to $y=2$ performs a full-twist with a block which consists of all the other points (see Figure 14).

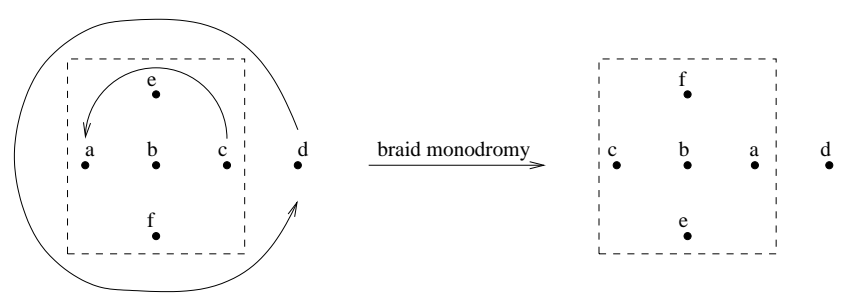

Figure 14. The action of the local braid monodromy

Now, we want to compute the induced relations of this singular point. According to van Kampen's Theorem, one should compute the g-base obtained by applying the action induced by the local braid monodromy of the singular point on the standard g-base. Since we have two complex points in the fiber before the action of the braid monodromy and after it, we have to start by rotating the two rightmost points by $90^{\circ}$ counterclockwise, for representing the two complex points (see Step (1) in Figure [15). Then, we move the two complex points to be over the second to the left real point (see Step (2) there). Now, we apply the action of the local braid monodromy (Steps (3) and (4)), and then we return the two new complex points to the right side, and return them to the real axis by rotating them clockwise by $90^{\circ}$ (Step (5), see Remark 2.9).

Now, by van Kampen's Theorem, we get the following induced relations from the new g-base (where $\left\{x_{1}, x_{2}, x_{3}, x_{4}, x_{5}, x_{6}\right\}$ are the generators of the standard g-base):

(1) $x_{1}=x_{5} x_{4} x_{3} x_{4}^{-1} x_{5}^{-1}$

(2) $x_{2}=x_{5} x_{4} x_{3} x_{2} x_{3}^{-1} x_{4}^{-1} x_{5}^{-1}$

(3) $x_{3}=x_{5} x_{4} x_{3} x_{2} x_{1} x_{2}^{-1} x_{3}^{-1} x_{4}^{-1} x_{5}^{-1}$

(4) $x_{4}=x_{5} x_{4} x_{3} x_{2} x_{1} x_{5} x_{4} x_{5}^{-1} x_{1}^{-1} x_{2}^{-1} x_{3}^{-1} x_{4}^{-1} x_{5}^{-1}$

(5) $x_{5}=x_{6}$

(6) $x_{6}=x_{6} x_{5} x_{4} x_{3} x_{2} x_{1} x_{5} x_{4} x_{5} x_{4}^{-1} x_{5}^{-1} x_{1}^{-1} x_{2}^{-1} x_{3}^{-1} x_{4}^{-1} x_{5}^{-1} x_{6}^{-1}$

Relation (2) can be written as: $x_{5} x_{4} x_{3} x_{2}=x_{2} x_{5} x_{4} x_{3}$. Using Relation (1), Relation (3) becomes: $x_{3} x_{5} x_{4} x_{3} x_{2} x_{5} x_{4}=x_{5} x_{4} x_{3} x_{2} x_{5} x_{4} x_{3}$.

By Relation (1) and some cancellations, Relation (4) becomes:

$$
x_{4}=x_{5} x_{4} x_{3} x_{2} x_{5} x_{4} x_{3} x_{4} x_{3}^{-1} x_{4}^{-1} x_{5}^{-1} x_{2}^{-1} x_{3}^{-1} x_{4}^{-1} x_{5}^{-1} .
$$

By Relation (3), we get:

$$
x_{4}=x_{3} x_{5} x_{4} x_{3} x_{2} x_{5} x_{4} x_{5}^{-1} x_{2}^{-1} x_{3}^{-1} x_{4}^{-1} x_{5}^{-1} x_{3}^{-1},
$$



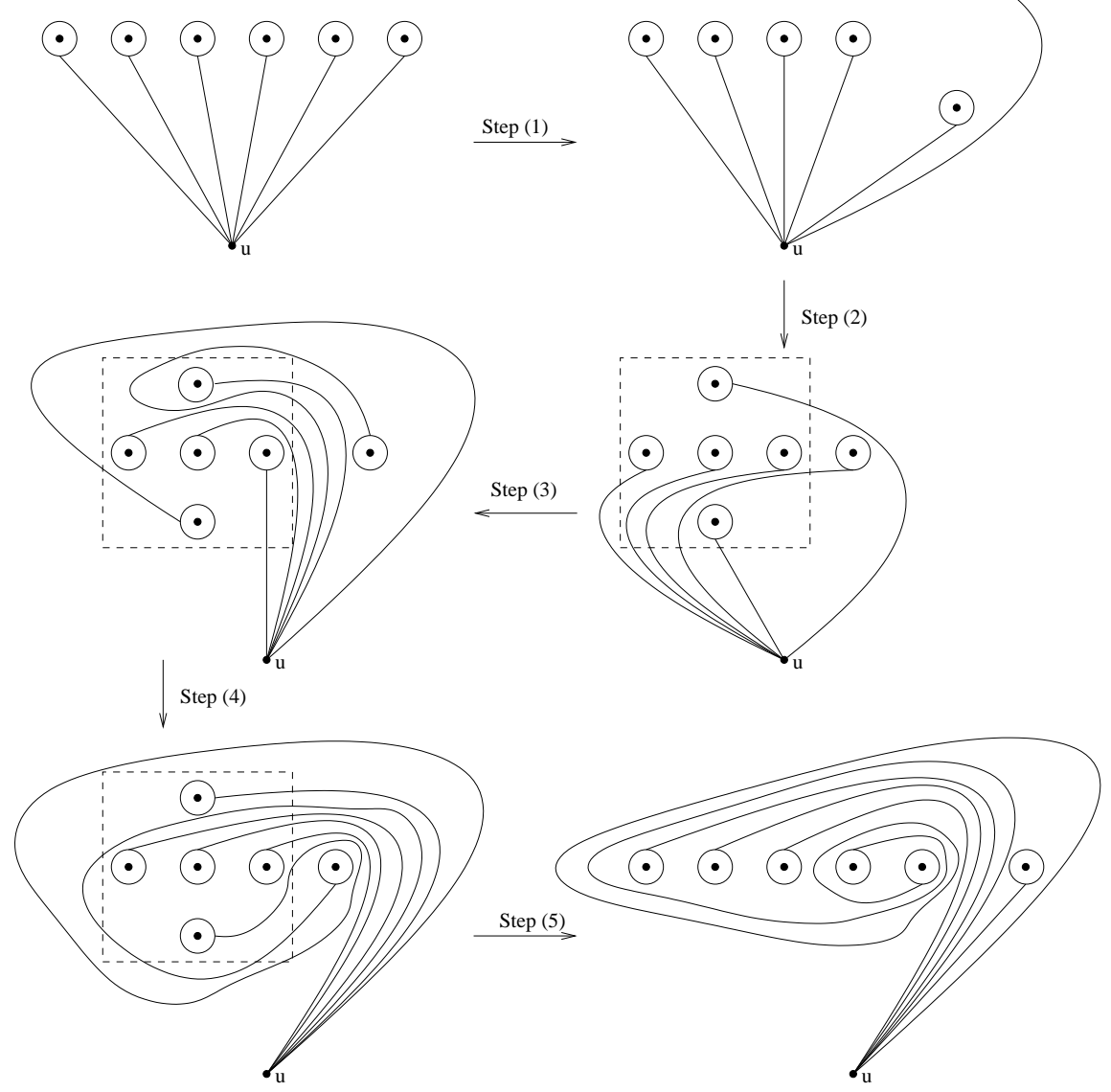

FiguRE 15. The g-base obtained from the standard gbase by the action of the local braid monodromy

and hence we have: $x_{4} x_{3} x_{5} x_{4} x_{3} x_{2} x_{5}=x_{3} x_{5} x_{4} x_{3} x_{2} x_{5} x_{4}$.

Now, we show that Relation (6) is redundant. First, this relation can be written as: $x_{5} x_{4} x_{3} x_{2} x_{1} x_{5} x_{4}=x_{4} x_{3} x_{2} x_{1} x_{5} x_{4} x_{5}$. By Relation (1) and some cancellations, we have: $x_{5} x_{4} x_{3} x_{2} x_{5} x_{4} x_{3}=x_{4} x_{3} x_{2} x_{5} x_{4} x_{3} x_{5}$. By Relation (3), we have: $x_{3} x_{5} x_{4} x_{3} x_{2} x_{5} x_{4}=x_{4} x_{3} x_{2} x_{5} x_{4} x_{3} x_{5}$. By Relation (2), this relation is equal to Relation (4), and hence Relation (6) is redundant.

Therefore, we get the following result for the set of relations for the singular point:

Corollary 2.18. The singular point presented locally by $x y\left(y+x^{2}\right)(y-$ $\left.x^{2}\right)=0$ has the following set of induced relations:

(1) $x_{5} x_{4} x_{3} x_{2}=x_{2} x_{5} x_{4} x_{3}$

(2) $x_{3} x_{5} x_{4} x_{3} x_{2} x_{5} x_{4}=x_{5} x_{4} x_{3} x_{2} x_{5} x_{4} x_{3}=x_{4} x_{3} x_{5} x_{4} x_{3} x_{2} x_{5}$ 
(3) $x_{1}=x_{5} x_{4} x_{3} x_{4}^{-1} x_{5}^{-1}$

(4) $x_{5}=x_{6}$

where $\left\{x_{1}, x_{2}, x_{3}, x_{4}, x_{5}, x_{6}\right\}$ are the generators of the standard $g$-base.

Since the Lefschetz diffeomorphism of the singular point is obtained by computing the action only on half of the unit circle (from $t=\frac{1}{2}$ to $t=1$ ), we have the following corollary:

Corollary 2.19. The Lefschetz diffeomorphism of the singular point presented locally by $x y\left(y+x^{2}\right)\left(y-x^{2}\right)=0$ is a $90^{\circ}$ counterclockwise rotation of the points correspond to $y=1,-1, i,-i$ around $y=0$, and then the point corresponds to $y=2$ do a half-twist with a block which consists of all the other points.

2.3.2. Two tangent conics with two intersecting lines. Although the following two types are almost a rotation of each other, we need them both, since the second type includes also "hidden" branch points inside the singularity, as we had in Section 2.2.3.

First type. The local equation of the singularity of the first type is $(2 x+y)(2 x-y)\left(y+x^{2}\right)\left(y-x^{2}\right)=0$ (see Figure 16).

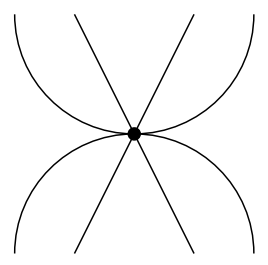

Figure 16. The singularity of $(2 x+y)(2 x-y)(y+$ $\left.x^{2}\right)\left(y-x^{2}\right)=0$ at $(0,0)$

Theorem 2.20. The local braid monodromy of the singularity presented locally by the equation $(2 x+y)(2 x-y)\left(y+x^{2}\right)\left(y-x^{2}\right)=0$ is: two points (correspond to $y=1$ and $y=-1$ ) do two counterclockwise full-twists and the two other points (corresponds to $y=2$ and $y=-2$ ) do a counterclockwise full-twist around them.

The singular point has the following induced relations:

$$
x_{4} x_{3} x_{2} x_{1}=x_{1} x_{4} x_{3} x_{2}=x_{3} x_{2} x_{1} x_{4} \quad ; \quad x_{4} x_{3} x_{2} x_{1} x_{3} x_{2}=x_{2} x_{4} x_{3} x_{2} x_{1} x_{3}
$$

where $\left\{x_{1}, x_{2}, x_{3}, x_{4}\right\}$ are the generators of the standard $g$-base.

The Lefschetz diffeomorphism of the singular point is a counterclockwise full-twist of the points $y=1$ and $y=-1$, and a counterclockwise half-twist of the points $y=2$ and $y=-2$ around the points $y=1,-1$. 


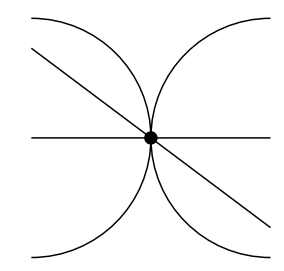

FiguRE 17. The singularity of $y(x+2 y)\left(y^{2}+x\right)\left(y^{2}-x\right)=$ 0 at $(0,0)$

Second type. The local equation of the singularity of the second type is $y(x+2 y)\left(y^{2}+x\right)\left(y^{2}-x\right)=0$ (see Figure 17).

There is a major difference between this type of singularity and the previous one: In this singularity there are two "hidden" branch points too (as in Section 2.2.3). That is, at any fiber, one has four real points and two complex points (i.e. complex level 2), and in each side of the singularity, the complex points belong to a different conic (since by the singularity, two real points become complex and two complex points become real).

For computing the braid monodromy in this case, we use the same trick we have already used in Section 2.3.1 the two lines in the middle, $x+2 y=0$ and $y=0$, can be thought for a moment as a "thick" line which is perpendicular to the tangent direction of the two tangent conics. We have already computed this case (Section 2.2.3): we got there that the braid monodromy is a $180^{\circ}$ counterclockwise rotation of four points around one fixed point at the origin. After observing this, we should add into account that the "thick" line stands for two lines. Hence, the fixed point at the origin is now decomposed into two close points, which are doing a counterclockwise full-twist (like a usual node). To summarize, we have:

Theorem 2.21. The local braid monodromy of the singularity presented locally by the equation $y(x+2 y)\left(y^{2}+x\right)\left(y^{2}-x\right)=0$ is: The four points correspond to $y=1,-1, i$ and $y=-i$ do a $180^{\circ}$ counterclockwise rotation, and the two points in the center do a counterclockwise full-twist (see Figure [18).

Now, we want to compute the induced relations of this singular point. By van Kampen's Theorem, we compute the g-base obtained by applying the action induced by the local braid monodromy of the singular point on the standard g-base. Since we have two complex points in the fiber before the action of the braid monodromy and after it, we have to start by rotating the two rightmost points by $90^{\circ}$ counterclockwise, for representing the two complex points (see Step (1) in Figure 19). Then, 


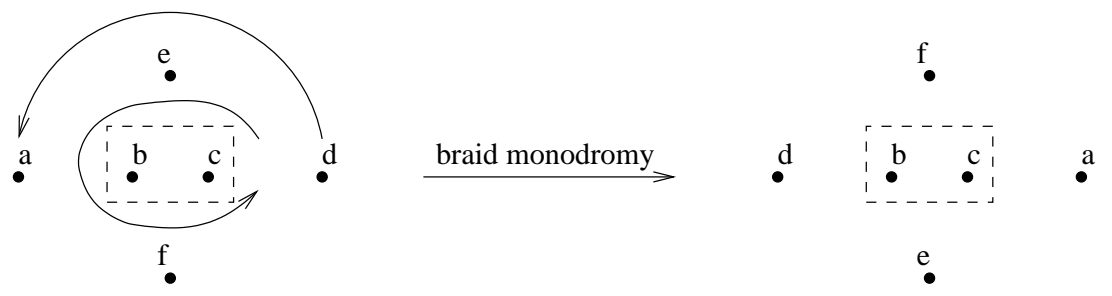

FiguRE 18. The action of the local braid monodromy

we move the two complex points to be over the two middle real points (see Step (2) there). Now, we apply the action of the local braid monodromy (Steps (3) and (4)), and then we return the two new complex points to the right side, and return them to the real axis by rotating them by $90^{\circ}$ clockwise (Step (5), see Remark 2.9).
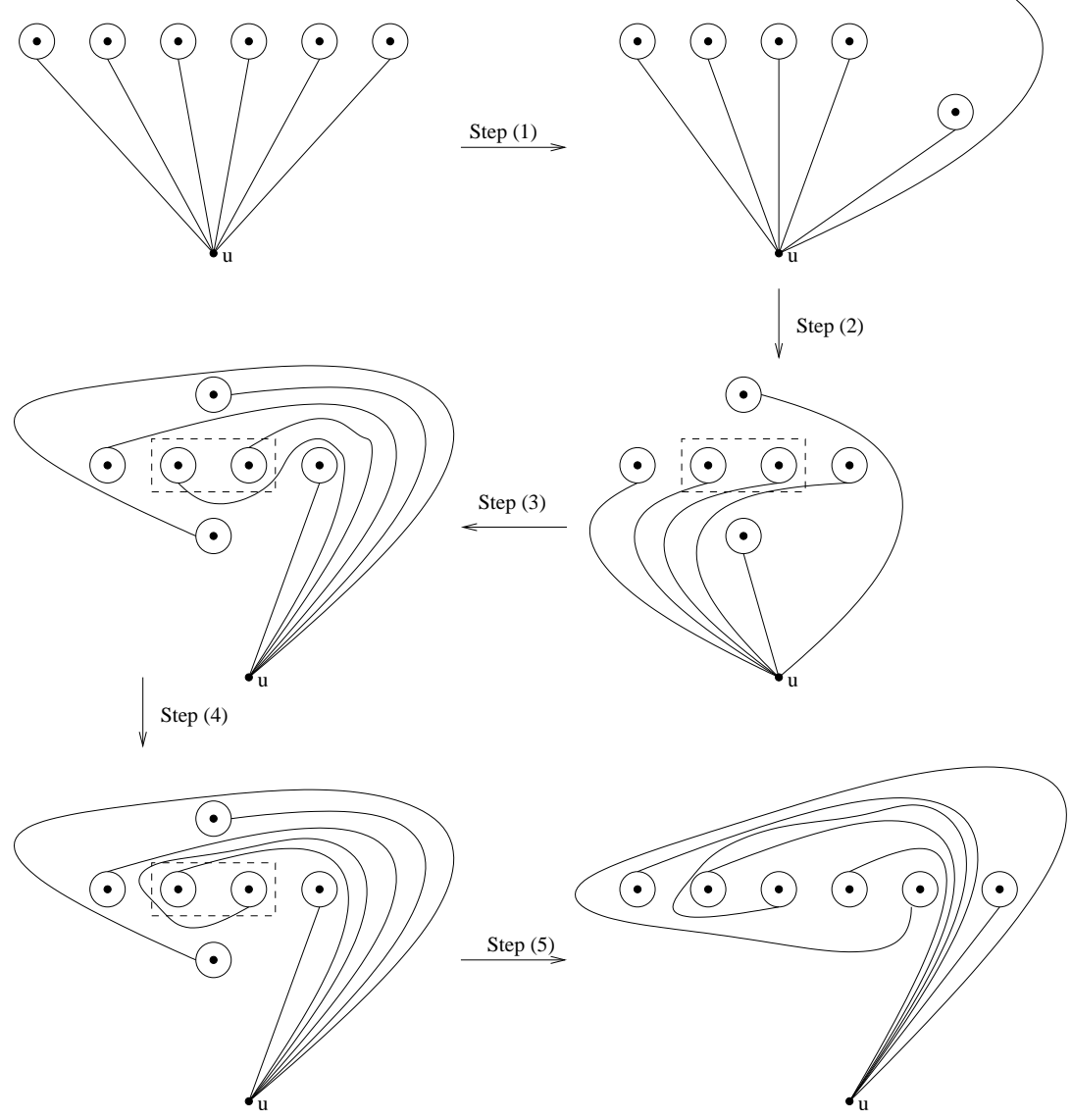

Figure 19. The g-base obtained from the standard gbase by the action of the local braid monodromy 
Now, by van Kampen's Theorem, we get the following induced relations from the new g-base (where $\left\{x_{1}, x_{2}, x_{3}, x_{4}, x_{5}, x_{6}\right\}$ are the generators of the standard g-base):

(1) $x_{1}=x_{5} x_{4} x_{5}^{-1}$

(2) $x_{2}=x_{5} x_{4} x_{3} x_{2} x_{3}^{-1} x_{4}^{-1} x_{5}^{-1}$

(3) $x_{3}=x_{5} x_{4} x_{3} x_{2} x_{3} x_{2}^{-1} x_{3}^{-1} x_{4}^{-1} x_{5}^{-1}$

(4) $x_{4}=x_{5} x_{4} x_{3} x_{2} x_{1} x_{2}^{-1} x_{3}^{-1} x_{4}^{-1} x_{5}^{-1}$

(5) $x_{5}=x_{6}$

(6) $x_{6}=x_{6} x_{5} x_{4} x_{3} x_{2} x_{1} x_{5} x_{1}^{-1} x_{2}^{-1} x_{3}^{-1} x_{4}^{-1} x_{5}^{-1} x_{6}^{-1}$

From Relation (2), we get $x_{5} x_{4} x_{3} x_{2}=x_{2} x_{5} x_{4} x_{3}$. Using this relation, Relation (3) becomes: $x_{3}=x_{2} x_{5} x_{4} x_{3} x_{4}^{-1} x_{5}^{-1} x_{2}^{-1}$, and hence $x_{2} x_{5} x_{4} x_{3}=$ $x_{3} x_{2} x_{5} x_{4}$. Using Relation (2) again, we get $x_{5} x_{4} x_{3} x_{2}=x_{3} x_{2} x_{5} x_{4}$.

By Relation (1), Relation (4) can be written: $x_{4} x_{5} x_{4} x_{3} x_{2} x_{5}=x_{5} x_{4} x_{3} x_{2} x_{5} x_{4}$

Now, we show that Relation (6) is redundant. Since $x_{5}=x_{6}$, we can simplify Relation (6) to the following form: $x_{5} x_{4} x_{3} x_{2} x_{1}=x_{4} x_{3} x_{2} x_{1} x_{5}$. Now, by Relation (1), we get: $x_{5} x_{4} x_{3} x_{2} x_{5} x_{4} x_{5}^{-1}=x_{4} x_{3} x_{2} x_{5} x_{4} x_{5}^{-1} x_{5}$. By some simplifications and Relation (3), we get Relation (4), and hence Relation (6) is redundant.

Therefore, we get the following result:

Corollary 2.22. The singular point presented locally by the equation $y(x+2 y)\left(y^{2}+x\right)\left(y^{2}-x\right)=0$ has the following set of relations:

(1) $x_{5} x_{4} x_{3} x_{2}=x_{2} x_{5} x_{4} x_{3}=x_{3} x_{2} x_{5} x_{4}$

(2) $x_{4} x_{5} x_{4} x_{3} x_{2} x_{5}=x_{5} x_{4} x_{3} x_{2} x_{5} x_{4}$

(3) $x_{1}=x_{5} x_{4} x_{5}^{-1}$

(4) $x_{5}=x_{6}$

where $\left\{x_{1}, x_{2}, x_{3}, x_{4}, x_{5}, x_{6}\right\}$ are the generators of the standard g-base.

If we delete the generators $x_{2}$ or $x_{3}$ which correspond to the lines $y=0$ or $x+2 y=0$ respectively, we get the set of relations as in Section 2.2 .3 as expected.

Since the Lefschetz diffeomorphism is a half of the action of the braid monodromy, we have that:

Corollary 2.23. The Lefschetz diffeomorphism of the singular point presented locally by $y(x+2 y)\left(y^{2}+x\right)\left(y^{2}-x\right)=0$ is a counterclockwise $90^{\circ}$ rotation of the four points around the center (which consists of two points), and then a counterclockwise half-twist of the two points in the center. 
$\pi_{1}$ OF ARRANGEMENTS WITH SINGULARITIES UP TO ORDER 6

\section{TWO CONICS WHICH ARE TANGENT TO EACH OTHER AT TWO POINTS}

In this section, we prove Proposition 1.1 which states that if $S$ is a curve in $\mathbb{C P}^{2}$ which composed of two tangent conics, then:

$$
\pi_{1}\left(\mathbb{C P}^{2}-S, *\right) \cong\left\langle x_{1}, x_{2} \mid\left(x_{1} x_{2}\right)^{2}=\left(x_{2} x_{1}\right)^{2}=e\right\rangle
$$

Proof of Proposition 1.1. Figure 20] shows a curve composed of two tangent conics.

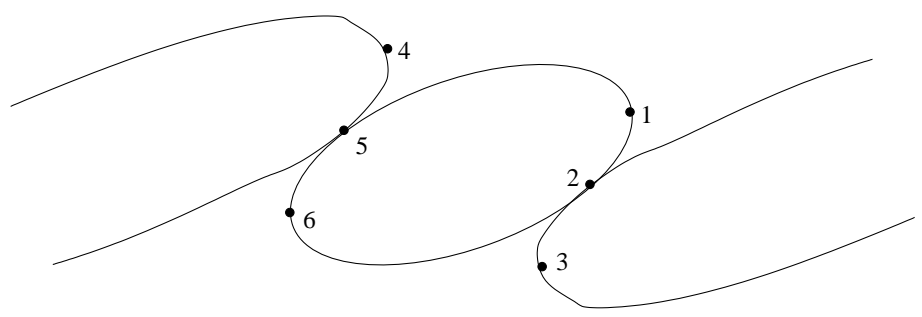

Figure 20. Two tangent conics

For computing the braid monodromy of this curve, we first have to compute the Lefschetz pairs of the singular points and to identify their types for applying the Moishezon-Teicher algorithm correctly (see [8]). In the following table, we summarize this data.

\begin{tabular}{|c|c|c|c|}
\hline $\mathrm{j}$ & $\lambda_{x_{j}}$ & $\epsilon_{x_{j}}$ & $\delta_{x_{j}}$ \\
\hline 1 & $P_{3}$ & 1 & $\Delta_{\mathbb{R} I_{2}}^{\frac{1}{2}}<3>$ \\
2 & $<2,3>$ & 4 & $\Delta^{2}<2,3>$ \\
3 & $<1,2>$ & 1 & $\Delta_{I_{2} \mathbb{R}}^{\frac{1}{2}}<1>$ \\
4 & $P_{3}$ & 1 & $\Delta_{\mathbb{R} I_{2}}^{\frac{1}{2}}<3>$ \\
5 & $<2,3>$ & 4 & $\Delta^{2}<2,3>$ \\
6 & $<1,2>$ & 1 & $\Delta_{I_{2} \mathbb{R}}^{\frac{1}{2}}<1>$ \\
\hline
\end{tabular}

By the Moishezon-Teicher algorithm, we get the skeletons related to the braid monodromy as shown in Figure 21.

By the van Kampen Theorem, we get the following presentation for the group $\pi_{1}\left(\mathbb{C P}^{2}-S\right)$ :

Generators: $\left\{x_{1}, x_{2}, x_{3}, x_{4}\right\}$.

Relations:

(1) $x_{4} x_{3} x_{2} x_{1}=e$ (projective relation)

(2) $x_{3}=x_{4}$

(3) $\left(x_{2} x_{4}\right)^{2}=\left(x_{4} x_{2}\right)^{2}$

(4) $x_{1}=x_{4} x_{2} x_{4}^{-1}$ 


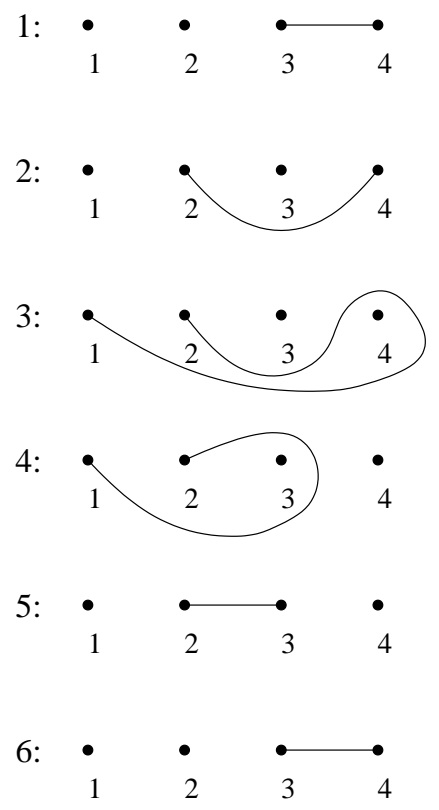

Figure 21. Skeletons for the braid monodromy of the curve in Proposition 1.1 (Figure 20)

(5) $x_{1}=x_{3} x_{2} x_{3}^{-1}$

(6) $\left(x_{2} x_{3}\right)^{2}=\left(x_{3} x_{2}\right)^{2}$

(7) $x_{3}=x_{4}$

It remains to show that this presentation is equivalent to the presentation of the group in the formulation of the proposition.

By Relation (2) (which is equal to Relation (7)), Relation (6) is equal to Relation (3) and Relation (5) is equal to Relation (4). Hence, Relations (5),(6) and (7) are redundant.

On the other hand, the first relation becomes $x_{3}^{2} x_{2} x_{1}=e$. By Relation (5), we have $x_{3}^{2} x_{2} x_{3} x_{2} x_{3}^{-1}=e$, which yields that: $\left(x_{3} x_{2}\right)^{2}=e$. We also get that $x_{1}$ is redundant, and therefore we get the requested presentation.

\section{Two TANGENT CONICS WITH AN ADDITIONAL LINE AND Proposition 1.2}

A simple tangency point can be presented locally as a tangency point between two smooth branches of the curve. We split our computations into two type of arrangements: arrangements with only simple tangency points and arrangements with other singular points. 
In the first subsection, we focus on arrangements with only simple tangency points, and then we deal with the other arrangement (second subsection).

4.1. An arrangement with a simple tangency point. In this section we prove that if $S$ is a curve in $\mathbb{C P}^{2}$ which composed of two tangent conics and one additional line which tangents to one of the conics and intersects the other (see Figure 22), then:

$$
\pi_{1}\left(\mathbb{C P}^{2}-S, *\right) \cong\left\langle x_{1}, x_{2} \mid\left(x_{1} x_{2}\right)^{2}=\left(x_{2} x_{1}\right)^{2}\right\rangle
$$

This configuration is the unique configuration of two tangent conics with an additional tangent line, which tangents to the conics in a simple tangency point (due to Remark [1.5).

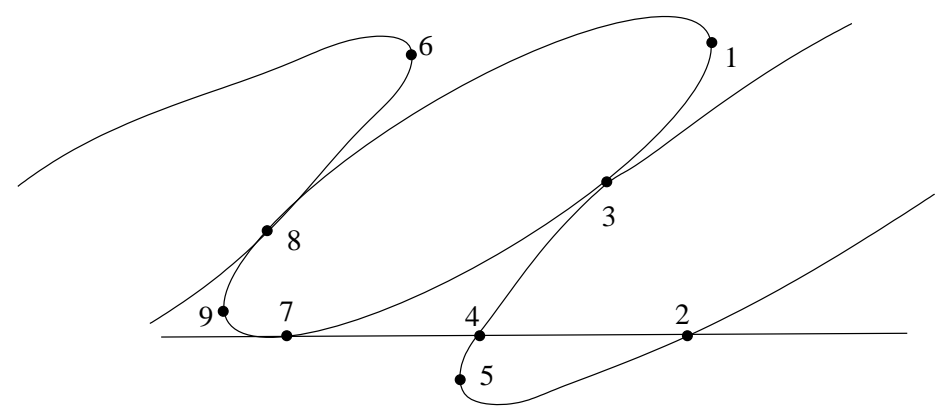

FiguRE 22. The conic-line arrangement with two tangent conics and a line which tangents in a simple tangency point

By the braid monodromy techniques and the van Kampen Theorem, we get the following presentation for $\pi_{1}\left(\mathbb{C P}^{2}-S\right)$ :

Generators: $\left\{x_{1}, x_{2}, x_{3}, x_{4}, x_{5}\right\}$.

Relations:

(1) $x_{5} x_{4} x_{3} x_{2} x_{1}=e$ (projective relation)

(2) $x_{4}=x_{5}$

(3) $x_{1} x_{2}=x_{2} x_{1}$

(4) $\left(x_{3} x_{5}\right)^{2}=\left(x_{5} x_{3}\right)^{2}$

(5) $\left(x_{2} x_{1} x_{2}^{-1}\right)\left(x_{5} x_{3} x_{5}^{-1}\right)=\left(x_{5} x_{3} x_{5}^{-1}\right)\left(x_{2} x_{1} x_{2}^{-1}\right)$

(6) $x_{2}=x_{5} x_{3} x_{5}^{-1}$

(7) $x_{1}^{-1} x_{2} x_{1}=x_{4} x_{3} x_{4}^{-1}$

(8) $\left(x_{2} x_{1} x_{2}^{-1} x_{5}\right)^{2}=\left(x_{5} x_{2} x_{1} x_{2}^{-1}\right)^{2}$

(9) $\left(x_{3} x_{4}\right)^{2}=\left(x_{4} x_{3}\right)^{2}$

(10) $x_{5}=x_{2} x_{1}^{-1} x_{2}^{-1} x_{4} x_{2} x_{1} x_{2}^{-1}$ 
By Relation (6), Relation (5) has the following form: $\left(x_{2} x_{1} x_{2}^{-1}\right) x_{2}=$ $x_{2}\left(x_{2} x_{1} x_{2}^{-1}\right)$. This is equal to $x_{1} x_{2}=x_{2} x_{1}$, which is already known by Relation (3). Hence, Relation (5) is redundant.

By Relation (3), Relation (7) is equal to $x_{2}=x_{4} x_{3} x_{4}^{-1}$, which is equivalent to Relation (6) by Relation (2). Hence, Relation (7) is redundant too.

By Relation (3) again, Relation (8) gets the form $\left(x_{1} x_{5}\right)^{2}=\left(x_{5} x_{1}\right)^{2}$, and Relation (10) is reduced to: $x_{5}=x_{1}^{-1} x_{4} x_{1}$. Since $x_{4}=x_{5}$, we have $x_{1} x_{5}=x_{5} x_{1}$. Hence, Relation (8) is redundant.

Relation (4) and Relation (9) are equal, since $x_{4}=x_{5}$.

Therefore, we have the following equivalent presentation:

Generators: $\left\{x_{1}, x_{2}, x_{3}, x_{5}\right\}$.

Relations:

(1) $x_{5}^{2} x_{3} x_{2} x_{1}=e$

(2) $x_{1} x_{2}=x_{2} x_{1}$

(3) $\left(x_{3} x_{5}\right)^{2}=\left(x_{5} x_{3}\right)^{2}$

(4) $x_{2}=x_{5} x_{3} x_{5}^{-1}$

(5) $x_{1} x_{5}=x_{5} x_{1}$

Substituting $x_{5} x_{3} x_{5}^{-1}$ for $x_{2}$ in Relation (1) and Relation (2), yields the relations $x_{5} x_{3} x_{5} x_{3} x_{1}=e$ and $x_{1} x_{3}=x_{3} x_{1}$ respectively. Hence, we get the following presentation:

Generators: $\left\{x_{1}, x_{3}, x_{5}\right\}$.

Relations:

(1) $x_{5} x_{3} x_{5} x_{3} x_{1}=e$

(2) $x_{1} x_{3}=x_{3} x_{1}$

(3) $\left(x_{3} x_{5}\right)^{2}=\left(x_{5} x_{3}\right)^{2}$

(4) $x_{1} x_{5}=x_{5} x_{1}$

By the first relation, $x_{1}=\left(x_{5} x_{3}\right)^{-2}$. Substituting it for $x_{1}$ in Relations (2) and (4) yields the relation $\left(x_{5} x_{3}\right)^{2}=\left(x_{3} x_{5}\right)^{2}$ twice, which is already known by Relation (3). Hence, Relations (2) and (4) are redundant.

So the final presentation is

$$
\left\langle x_{3}, x_{5} \mid\left(x_{3} x_{5}\right)^{2}=\left(x_{5} x_{3}\right)^{2}\right\rangle
$$

which appears in Proposition 1.2 as needed.

Remark 4.1. This fundamental group was computed independently by Degtyarev too (see [2, Section 3.3.5]). 
4.2. Arrangements with other singular points. Apart from the previous case, we have four more possibilities to locate a line into a configuration of two tangent conics:

(1) The line intersects the conic transversally.

(2) The line passes through one of the tangency points between the two conics, but it is not tangent to the conics (see Figure 23).

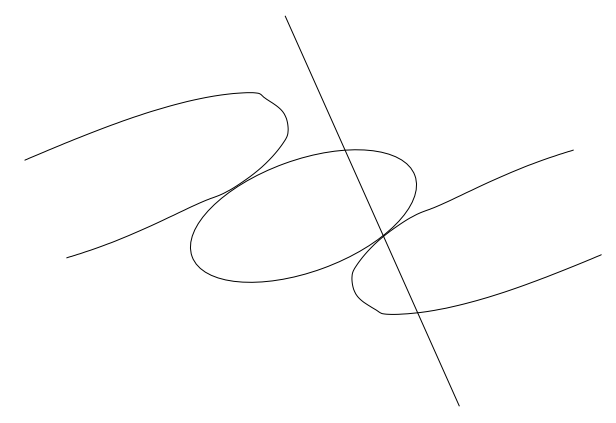

Figure 23. The line passes through the tangency point but it is not tangent

(3) The line passes through one of the tangency points between the two conics, and it is tangent to the conics (see Figure 24).

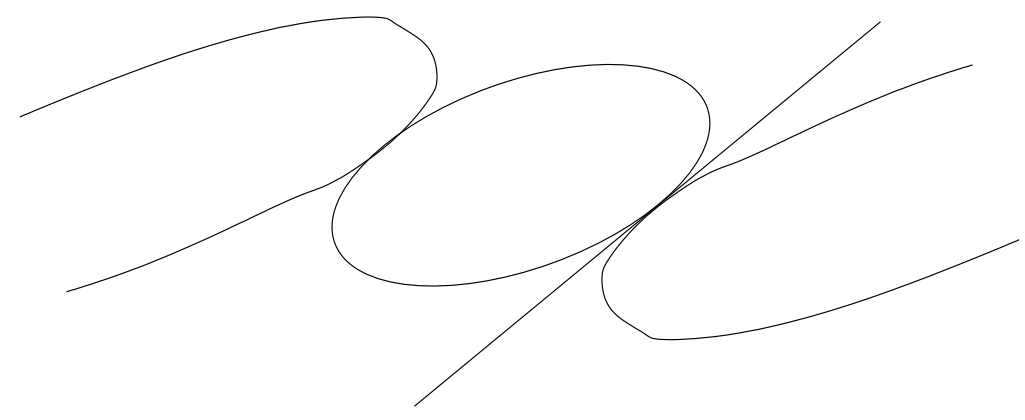

Figure 24. The line passes through the tangency point and it is tangent

(4) The line passes through the two tangency points (see Figure 251).

Based on Proposition 1.1] and [3], the fundamental group of the complement of the first case is

$$
\mathbb{Z} \oplus\left\langle x_{1}, x_{2} \mid\left(x_{1} x_{2}\right)^{2}=\left(x_{2} x_{1}\right)^{2}=e\right\rangle,
$$

where $e$ is the identity element of the group.

In the following subsections, we compute the third and the fourth cases, whence the result of the second case turns out to be isomorphic to the third case. 


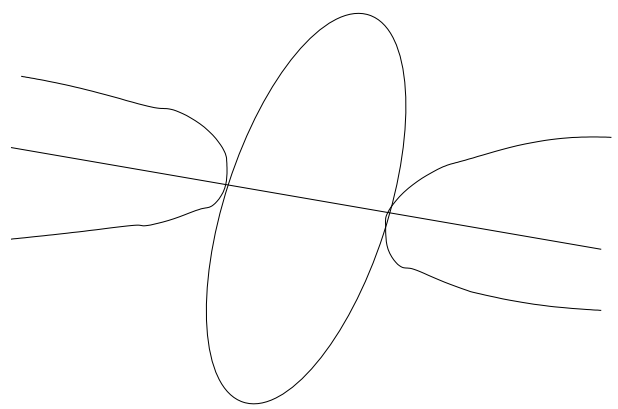

Figure 25. The line passes through the two tangency points

4.2.1. Third case. Using braid monodromy techniques and the van Kampen theorem, we get the following presentation for the fundamental group of the complement of the curve which appear in Figure 24 .

Generators: $\left\{x_{1}, x_{2}, x_{3}, x_{4}, x_{5}\right\}$.

Relations:

(1) $x_{5} x_{4} x_{3} x_{2} x_{1}=e$ (projective relation)

(2) $x_{4}=x_{5}$

(3) $\left(x_{5} x_{3} x_{2}\right)^{2}=\left(x_{3} x_{2} x_{5}\right)^{2}=\left(x_{2} x_{5} x_{3}\right)^{2}$

(4) $x_{1}=x_{5} x_{3} x_{2} x_{3}^{-1} x_{5}^{-1}$

(5) $x_{1}=x_{4} x_{2} x_{4}^{-1}$

(6) $\left(x_{2} x_{4}\right)^{2}=\left(x_{4} x_{2}\right)^{2}$

(7) $x_{3}^{-1} x_{4} x_{3}=x_{5}$

By Relations (2) and (5), Relation (4) becomes $x_{4} x_{3} x_{2} x_{3}^{-1} x_{4}^{-1}=$ $x_{4} x_{2} x_{4}^{-1}$, and hence $x_{2} x_{3}=x_{3} x_{2}$

By Relation (2), we get from Relation (6) that $x_{3} x_{4}=x_{4} x_{3}$.

Using Relations (2) and (5) again, the first relation becomes: $x_{4} x_{4} x_{3} x_{2} x_{4} x_{2} x_{4}^{-1}=$ $e$. By some cancellations and Relation (6), we get: $x_{3}=\left(x_{2} x_{4}\right)^{-2}$.

Hence, we get the following equivalent presentation:

Generators: $\left\{x_{2}, x_{3}, x_{4}\right\}$.

Relations:

(1) $x_{3}=\left(x_{2} x_{4}\right)^{-2}$

(2) $\left(x_{4} x_{3} x_{2}\right)^{2}=\left(x_{3} x_{2} x_{4}\right)^{2}=\left(x_{2} x_{4} x_{3}\right)^{2}$

(3) $x_{2} x_{3}=x_{3} x_{2}$

(4) $\left(x_{2} x_{4}\right)^{2}=\left(x_{4} x_{2}\right)^{2}$

(5) $x_{4} x_{3}=x_{3} x_{4}$

By Relation (1) and (4), Relations (3),(5) and the right equation of Relation (2) become trivial. 
For the left equation of Relation (2), be Relation (1) we substitute $x_{3}$ by $\left(x_{2} x_{4}\right)^{-2}$, to get:

$$
x_{4}\left(x_{2} x_{4}\right)^{-2} x_{2} x_{4}\left(x_{2} x_{4}\right)^{-2} x_{2}=\left(x_{2} x_{4}\right)^{-2} x_{2} x_{4}\left(x_{2} x_{4}\right)^{-2} x_{2} x_{4} .
$$

By cancellations, we get:

$$
x_{4}\left(x_{2} x_{4}\right)^{-3} x_{2}=\left(x_{2} x_{4}\right)^{-2}
$$

which becomes trivial by Relation (4) and hence it is redundant.

Therefore, we get the following final presentation:

$$
\pi_{1}\left(\mathbb{C P}^{2}-S\right) \cong\left\langle x_{2}, x_{4} \mid\left(x_{2} x_{4}\right)^{2}=\left(x_{4} x_{2}\right)^{2}\right\rangle
$$

4.2.2. Fourth case. Using braid monodromy techniques and the van Kampen theorem, we get the following presentation for the fundamental group of the complement of the curve which appear in Figure 25.

Generators: $\left\{x_{1}, x_{2}, x_{3}, x_{4}, x_{5}\right\}$.

Relations:

(1) $x_{5} x_{4} x_{3} x_{2} x_{1}=e$ (projective relation)

(2) $x_{4}=x_{5}$

(3) $x_{1}=x_{4} x_{3} x_{4}^{-1}$

(4) $x_{2} x_{4} x_{3}=x_{4} x_{3} x_{2}$

(5) $x_{4} x_{3} x_{2} x_{4} x_{3}=x_{3} x_{2} x_{4} x_{3} x_{4}$

(6) $x_{1}=x_{2}^{-1} x_{3}^{-1} x_{4}^{-1} x_{3} x_{4} x_{3} x_{2}$

(7) $x_{2}^{-1} x_{3}^{-1} x_{4} x_{3} x_{2}=x_{1} x_{5} x_{1}^{-1}$

(8) $x_{2} x_{1} x_{5}=x_{1} x_{5} x_{2}$

(9) $x_{1} x_{5} x_{2} x_{1} x_{5}=x_{5} x_{2} x_{1} x_{5} x_{1}$

By Relations (2) and (3) we replace $x_{5}$ and $x_{1}$ by $x_{4}$ and $x_{4} x_{3} x_{4}^{-1}$ respectively. By these replacements, Relations (4) and (8) become equal. Moreover, Relations (5) and (9) are the same (using Relation (4)). So we get the following presentation:

Generators: $\left\{x_{1}, x_{2}, x_{3}, x_{4}, x_{5}\right\}$.

Relations:

(1) $x_{4}^{2} x_{3} x_{2} x_{4} x_{3} x_{4}^{-1}=e$

(2) $x_{2} x_{4} x_{3}=x_{4} x_{3} x_{2}$

(3) $x_{4} x_{3} x_{2} x_{4} x_{3}=x_{3} x_{2} x_{4} x_{3} x_{4}$

(4) $x_{4} x_{3} x_{4}^{-1}=x_{2}^{-1} x_{3}^{-1} x_{4}^{-1} x_{3} x_{4} x_{3} x_{2}$

(5) $x_{2}^{-1} x_{3}^{-1} x_{4} x_{3} x_{2}=x_{4} x_{3} x_{4} x_{3}^{-1} x_{4}^{-1}$

Relation (1) can be simplified (using Relation (2)) to the following form: $x_{2} x_{4} x_{3} x_{4} x_{3}=e$. Hence, we have: $x_{2}=\left(x_{4} x_{3}\right)^{-2}$. Using this, all the other relations become trivial (by simple computations).

Hence, the resulting group is the free group with two generators. 
Remark 4.2. The above two results were computed independently by Degtyarev too (see [2]).

\section{Two TANGENT CONICS WITH TWO ADDITIONAL LINES AND Proposition 1.3}

As in the previous section, we divide our treatment into two cases: We first focus on arrangements with only simple tangency points (first subsection), and then we deal with the other arrangement (second subsection).

5.1. Arrangements with simple tangency points. In this section, we present the fundamental groups of the complement of the two different possibilities for curves with two tangent conics and two additional lines which are tangent in only simple tangency points: the case where each line is tangent a different conic, and the case where the two lines are tangent to the same conic.

Theorem 5.1. Let $S$ be a curve in $\mathbb{C P}^{2}$ composed of two tangent conics and two additional lines, where each line is tangent to a different conic (see Figure 26), then:

$$
\pi_{1}\left(\mathbb{C P}^{2}-S, *\right) \cong\left\langle x_{1}\right\rangle \oplus\left\langle x_{2}, x_{3} \mid\left(x_{2} x_{3}\right)^{2}=\left(x_{3} x_{2}\right)^{2}\right\rangle
$$

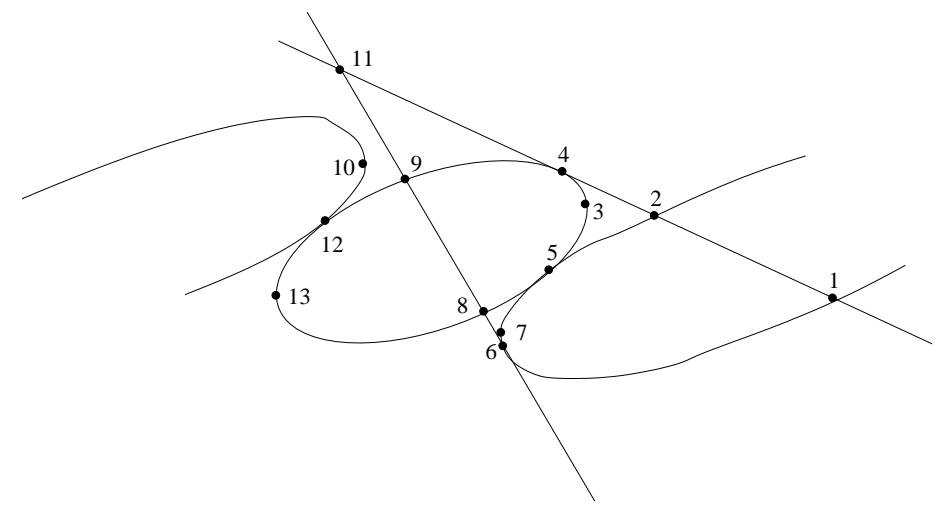

Figure 26. The conic-line arrangement where each line is tangent to a different conic

Theorem 5.2. Let $S$ be a curve in $\mathbb{C P}^{2}$ composed of two tangent conics and two additional lines, which are both tangent to the same conic (see Figure 27, then:

$\pi_{1}\left(\mathbb{C P}^{2}-S, *\right) \cong\left\langle\begin{array}{c|c}x_{1}, x_{2}, x_{3} & \left(x_{2} x_{3}\right)^{2}=\left(x_{3} x_{2}\right)^{2},\left(x_{1} x_{3}\right)^{2}=\left(x_{3} x_{1}\right)^{2}, \\ {\left[x_{1}, x_{2}\right]=\left[x_{2}, x_{3} x_{1} x_{3}^{-1}\right]=e}\end{array}\right\rangle$ 


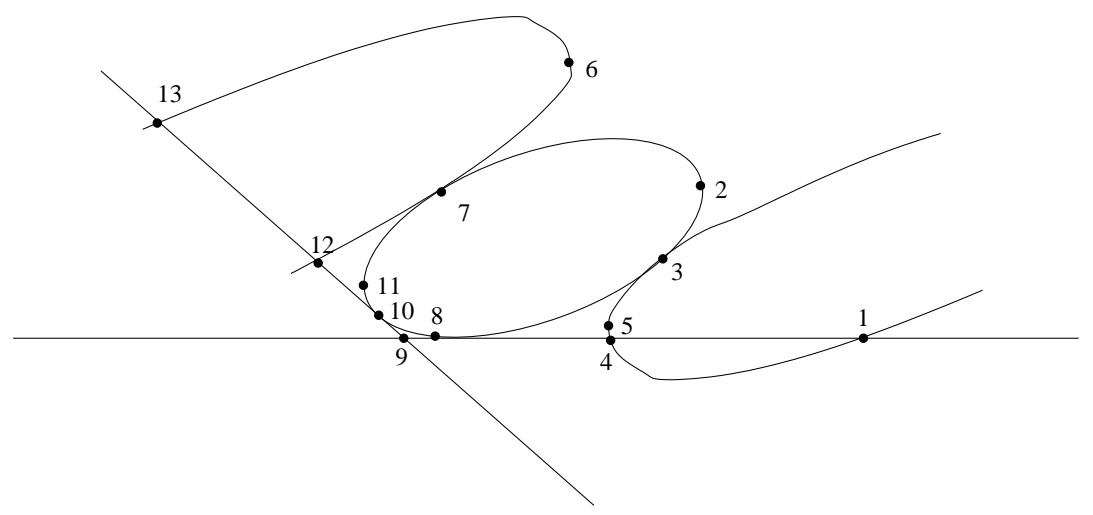

FiguRE 27. The conic-line arrangement where both lines are tangent to the same conic

The proofs of the above theorems are similar to the ones in Section 4.1

5.2. Arrangements with other singularities. Apart from the two previous cases of arrangements with only simple tangency points, we have 16 more possibilities to locate two lines into a configuration of two tangent conics.

In the following subsections, we compute only the cases which turn out to have nonisomorphic fundamental groups of the complements. In the appendix, we list all the 16 possibilities with the corresponding fundamental groups of their complements.

5.2.1. First case. In this subsection, we compute the fundamental group of the complement of the curve $S$ presented in Figure 28

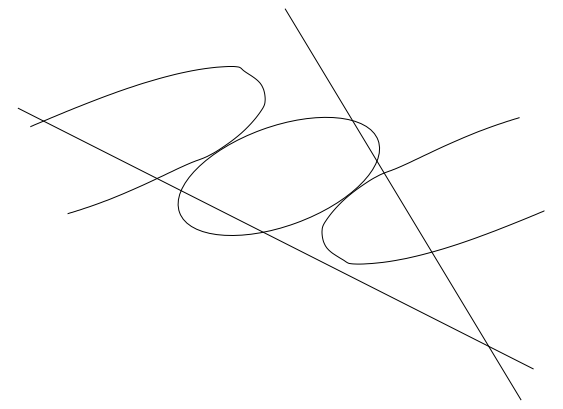

Figure 28. First case

Based on Proposition 1.1] and 3], the fundamental group of the complement of this case is

$$
\mathbb{Z}^{2} \oplus\left\langle x_{1}, x_{2} \mid\left(x_{1} x_{2}\right)^{2}=\left(x_{2} x_{1}\right)^{2}=e\right\rangle,
$$


where $e$ is the identity element of the group.

5.2.2. Second case. In this subsection, we compute the fundamental group of the complement of the curve $S$ presented in Figure 29.

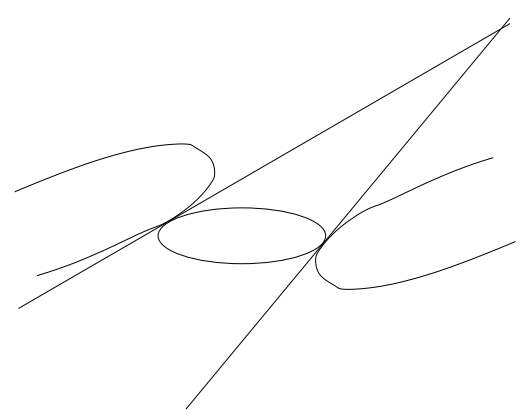

Figure 29. Second case

Using braid monodromy techniques and the van Kampen theorem, we get the following presentation for the fundamental group of the complement:

Generators: $\left\{x_{1}, x_{2}, x_{3}, x_{4}, x_{5}, x_{6}\right\}$.

Relations:

(1) $x_{6} x_{5} x_{4} x_{3} x_{2} x_{1}=e$ (projective relation)

(2) $x_{3} x_{5}^{-1} x_{6} x_{5}=x_{5}^{-1} x_{6} x_{5} x_{3}$

(3) $x_{3}^{-1} x_{4} x_{3}=x_{5}^{-1} x_{6}^{-1} x_{5} x_{6} x_{5}$

(4) $\left(x_{5} x_{5}^{-1} x_{6} x_{5} x_{2}\right)^{2}=\left(x_{2} x_{5} x_{5}^{-1} x_{6} x_{5}\right)^{2}=\left(x_{5}^{-1} x_{6} x_{5} x_{2} x_{5}\right)^{2}$

(5) $x_{1}=x_{6} x_{5} x_{2} x_{5}^{-1} x_{6}^{-1}$

(6) $x_{1}=x_{4} x_{3} x_{2} x_{3}^{-1} x_{4}^{-1}$

(7) $\left(x_{4} x_{3} x_{2}\right)^{2}=\left(x_{2} x_{4} x_{3}\right)^{2}=\left(x_{3} x_{2} x_{4}\right)^{2}$

(8) $x_{4}=x_{5}$

In the first step, we use Relation (8) to substitute $x_{4}$ for $x_{5}$ everywhere. Moreover, since Relations (5) and (6) are both equal to $x_{1}$, we can write the following equality:

$$
x_{6} x_{4} x_{2} x_{4}^{-1} x_{6}^{-1}=x_{4} x_{3} x_{2} x_{3}^{-1} x_{4}^{-1}
$$

Using Relation (5) again, we have that the projective relation gets the following form:

$$
x_{6} x_{4}^{2} x_{3} x_{2} x_{6} x_{4} x_{2} x_{4}^{-1} x_{6}^{-1}=e
$$

Hence, the generator $x_{1}$ is redundant.

So we get the following equivalent presentation:

Generators: $\left\{x_{2}, x_{3}, x_{4}, x_{6}\right\}$.

Relations: 
(1) $x_{6} x_{4}^{2} x_{3} x_{2} x_{6} x_{4} x_{2} x_{4}^{-1} x_{6}^{-1}=e$

(2) $x_{3} x_{4}^{-1} x_{6} x_{4}=x_{4}^{-1} x_{6} x_{4} x_{3}$

(3) $x_{3}^{-1} x_{4} x_{3}=x_{4}^{-1} x_{6}^{-1} x_{4} x_{6} x_{4}$

(4) $\left(x_{6} x_{4} x_{2}\right)^{2}=\left(x_{2} x_{6} x_{4}\right)^{2}=\left(x_{4}^{-1} x_{6} x_{4} x_{2} x_{4}\right)^{2}$

(5) $x_{6} x_{4} x_{2} x_{4}^{-1} x_{6}^{-1}=x_{4} x_{3} x_{2} x_{3}^{-1} x_{4}^{-1}$

(6) $\left(x_{4} x_{3} x_{2}\right)^{2}=\left(x_{2} x_{4} x_{3}\right)^{2}=\left(x_{3} x_{2} x_{4}\right)^{2}$

From the first relation, we have that: $x_{4} x_{3} x_{2} x_{6} x_{4} x_{2}=e$, and hence:

$$
x_{3}=x_{4}^{-1} x_{2}^{-1} x_{4}^{-1} x_{6}^{-1} x_{2}^{-1}
$$

Now, we replace $x_{3}$ by $x_{4}^{-1} x_{2}^{-1} x_{4}^{-1} x_{6}^{-1} x_{2}^{-1}$, in any place it appears.

Relation (2) gets the following form:

$$
x_{4}^{-1} x_{2}^{-1} x_{4}^{-1} x_{6}^{-1} x_{2}^{-1} x_{4}^{-1} x_{6} x_{4}=x_{4}^{-1} x_{6} x_{4} x_{4}^{-1} x_{2}^{-1} x_{4}^{-1} x_{6}^{-1} x_{2}^{-1}
$$

By some reorderings, we get that:

$$
\left(x_{6} x_{4} x_{2}\right)^{2}=\left(x_{4} x_{2} x_{6}\right)^{2} .
$$

Relation (3) can now be written as:

$$
x_{2} x_{6} x_{4} x_{2} x_{4} x_{4} x_{4}^{-1} x_{2}^{-1} x_{4}^{-1} x_{6}^{-1} x_{2}^{-1}=x_{4}^{-1} x_{6}^{-1} x_{4} x_{6} x_{4}
$$

By some cancellations, we get that: $\left(x_{6} x_{4} x_{2}\right)^{2} x_{4}=x_{4}\left(x_{6} x_{4} x_{2}\right)^{2}$. By Relation (2), this is equal to: $\left(x_{2} x_{6} x_{4}\right)^{2}=\left(x_{6} x_{4} x_{2}\right)^{2}$, which is known (Relation (4)), and hence Relation (3) is redundant.

Relation (5) can be written:

$$
x_{6} x_{4} x_{2} x_{4}^{-1} x_{6}^{-1}=x_{4} x_{4}^{-1} x_{2}^{-1} x_{4}^{-1} x_{6}^{-1} x_{2}^{-1} x_{2} x_{2} x_{6} x_{4} x_{2} x_{4} x_{4}^{-1}
$$

By some cancellations and reorderings, this is equal to:

$$
\left(x_{6} x_{4} x_{2}\right)^{2}=\left(x_{2} x_{6} x_{4}\right)^{2}
$$

which is known (from Relation (2)), and hence this relation is redundant too.

By similar computations, Relation (6) can be written as:

$$
\left(x_{2} x_{6} x_{4}\right)^{2}=\left(x_{6} x_{4} x_{2}\right)^{2}=\left(x_{4} x_{2} x_{6}\right)^{2}
$$

which is known (Relations (2) and (4)), and hence Relation (6) is redundant too.

The right part of Relation (4) can be written as:

$$
\left(x_{2} x_{6} x_{4}\right)^{2}=x_{4}^{-1}\left(x_{6} x_{4} x_{2}\right)^{2} x_{4}
$$

which is known by Relation (2), and therefore it is redundant too.

Hence, we get the following equivalent presentation:

$$
\left\langle x_{2}, x_{4}, x_{6} \mid\left(x_{6} x_{4} x_{2}\right)^{2}=\left(x_{2} x_{6} x_{4}\right)^{2}=\left(x_{4} x_{2} x_{6}\right)^{2}\right\rangle
$$

as needed. 
5.2.3. Third case. In this subsection, we compute the fundamental group of the complement of the curve $S$ presented in Figure 30.

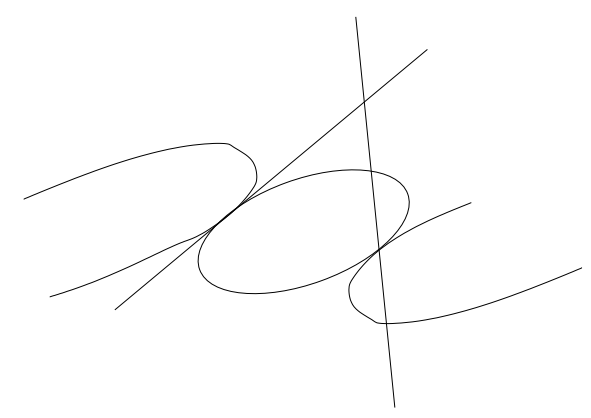

FiguRE 30. Third case

Using braid monodromy techniques and the van Kampen theorem, we get the following presentation for the fundamental group of the complement:

Generators: $\left\{x_{1}, x_{2}, x_{3}, x_{4}, x_{5}, x_{6}\right\}$.

Relations:

(1) $x_{6} x_{5} x_{4} x_{3} x_{2} x_{1}=e$ (projective relation)

(2) $x_{4}=x_{5}$

(3) $x_{1} x_{2}=x_{2} x_{1}$

(4) $x_{2} x_{1} x_{2}^{-1} x_{5} x_{3}=x_{5} x_{3} x_{2} x_{1} x_{2}^{-1}$

(5) $x_{5} x_{3} x_{2} x_{1} x_{2}^{-1} x_{5} x_{3}=x_{3} x_{5} x_{3} x_{2} x_{1} x_{2}^{-1} x_{5}$

(6) $x_{1} x_{4}=x_{4} x_{1}$

(7) $x_{1} x_{6}=x_{6} x_{1}$

(8) $x_{2}=x_{5} x_{3} x_{5}^{-1}$

(9) $x_{2}=x_{6} x_{4} x_{3} x_{4}^{-1} x_{6}^{-1}$

(10) $\left(x_{6} x_{5} x_{4} x_{5}^{-1} x_{6}^{-1} x_{6} x_{5} x_{3} x_{5}^{-1}\right)^{2}=\left(x_{5} x_{3} x_{5}^{-1} x_{6} x_{5} x_{4} x_{5}^{-1} x_{6}^{-1} x_{6}\right)^{2}=$ $\left(x_{6} x_{5} x_{3} x_{5}^{-1} x_{6} x_{5} x_{4} x_{5}^{-1} x_{6}^{-1}\right)^{2}$

(11) $x_{4}=x_{6} x_{5} x_{6}^{-1}$

Using Relations (2) and (8), we can cancel generators $x_{2}$ and $x_{5}$, and replace them by $x_{4} x_{3} x_{4}^{-1}$ and $x_{4}$ respectively. So we get the following simplified presentation:

Generators: $\left\{x_{1}, x_{3}, x_{4}, x_{6}\right\}$.

Relations:

(1) $x_{6} x_{4} x_{4} x_{3} x_{4} x_{3} x_{4}^{-1} x_{1}=e$

(2) $x_{1} x_{4} x_{3} x_{4}^{-1}=x_{4} x_{3} x_{4}^{-1} x_{1}$

(3) $x_{4} x_{3} x_{4}^{-1} x_{1} x_{4}=x_{4} x_{3} x_{4} x_{3} x_{4}^{-1} x_{1} x_{4} x_{3}^{-1} x_{4}^{-1}$

(4) $x_{4} x_{3} x_{4} x_{3} x_{4}^{-1} x_{1} x_{4}=x_{3} x_{4} x_{3} x_{4} x_{3} x_{4}^{-1} x_{1} x_{4} x_{3}^{-1}$

(5) $x_{1} x_{4}=x_{4} x_{1}$ 
(6) $x_{1} x_{6}=x_{6} x_{1}$

(7) $x_{4} x_{3} x_{4}^{-1}=x_{6} x_{4} x_{3} x_{4}^{-1} x_{6}^{-1}$

(8) $\left(x_{6} x_{4} x_{6}^{-1} x_{6} x_{4} x_{3} x_{4}^{-1}\right)^{2}=\left(x_{4} x_{3} x_{4}^{-1} x_{6} x_{4} x_{6}^{-1} x_{6}\right)^{2}=\left(x_{6} x_{4} x_{3} x_{4}^{-1} x_{6} x_{4} x_{6}^{-1}\right)^{2}$

(9) $x_{4}=x_{6} x_{4} x_{6}^{-1}$

By Relation (5), Relation (2) is reduced to $x_{4} x_{3}=x_{3} x_{1}$. By this relation and Relation (5) again, Relation (3) can be simplified to the trivial relation, and hence it is redundant. Moreover, Relation (4) gets the following form: $\left(x_{4} x_{3}\right)^{2}=\left(x_{3} x_{4}\right)^{2}$.

By Relation (9), we can simplify Relations (7) and (8): they get the following forms respectively: $x_{3} x_{6}=x_{6} x_{3}$ and $\left(x_{4} x_{6} x_{4} x_{3} x_{4}^{-1}\right)^{2}=$ $\left(x_{4} x_{3} x_{6}\right)^{2}=\left(x_{6} x_{4} x_{3}\right)^{2}$.

Hence, we get the following simplified presentation:

Generators: $\left\{x_{1}, x_{3}, x_{4}, x_{6}\right\}$.

Relations:

(1) $x_{6} x_{4} x_{4} x_{3} x_{4} x_{3} x_{4}^{-1} x_{1}=e$

(2) $x_{1} x_{3}=x_{3} x_{1}$

(3) $\left(x_{4} x_{3}\right)^{2}=\left(x_{3} x_{4}\right)^{2}$

(4) $x_{1} x_{4}=x_{4} x_{1}$

(5) $x_{1} x_{6}=x_{6} x_{1}$

(6) $x_{3} x_{6}=x_{6} x_{3}$

(7) $\left(x_{4} x_{6} x_{4} x_{3} x_{4}^{-1}\right)^{2}=\left(x_{4} x_{3} x_{6}\right)^{2}=\left(x_{6} x_{4} x_{3}\right)^{2}$

(8) $x_{4} x_{6}=x_{6} x_{4}$

Since $x_{6}$ commutes with all the other generators, Relation (7) is reduced to $\left(x_{4}^{2} x_{3} x_{4}^{-1}\right)^{2}=\left(x_{4} x_{3}\right)^{2}=\left(x_{4} x_{3}\right)^{2}$ The right equation is known (Relation (3)), so we have to simplify only the left equation. By some simplifications, we get $x_{4} x_{3} x_{4} x_{3}=x_{3} x_{4} x_{3} x_{4}$ which is known too, and hence this relation is redundant.

Now, by Relation (1), we have that $x_{6}=x_{1}^{-1}\left(x_{4} x_{3}\right)^{-2}$. So we can replace $x_{6}$ by $x_{1}^{-1}\left(x_{4} x_{3}\right)^{-2}$ in any place it appears.

Relation (5) gets the form: $x_{1} x_{1}^{-1}\left(x_{4} x_{3}\right)^{-2}=x_{1}^{-1}\left(x_{4} x_{3}\right)^{-2} x_{1}$ Since $x_{1}$ commutes with $x_{3}$ and $x_{4}$, this relation becomes trivial.

Relations (6) and (8) get the following forms respectively: $x_{3} x_{1}^{-1}\left(x_{4} x_{3}\right)^{-2}=$ $x_{1}^{-1}\left(x_{4} x_{3}\right)^{-2} x_{3}$ and $x_{4} x_{1}^{-1}\left(x_{4} x_{3}\right)^{-2}=x_{1}^{-1}\left(x_{4} x_{3}\right)^{-2} x_{4}$. Again, by using Relations (2), (3) and (4), these relations become trivial.

Hence, we get the following simplified presentation:

Generators: $\left\{x_{1}, x_{3}, x_{4}\right\}$.

Relations:

(1) $x_{1} x_{3}=x_{3} x_{1}$

(2) $\left(x_{4} x_{3}\right)^{2}=\left(x_{3} x_{4}\right)^{2}$

(3) $x_{1} x_{4}=x_{4} x_{1}$ 
This group is obviously isomorphic to:

$$
\left\langle x_{1}\right\rangle \oplus\left\langle x_{3}, x_{4} \mid\left(x_{4} x_{3}\right)^{2}=\left(x_{3} x_{4}\right)^{2}\right\rangle
$$

as needed.

5.2.4. Fourth case. In this subsection, we compute the fundamental group of the complement of the curve $S$ presented in Figure 31.

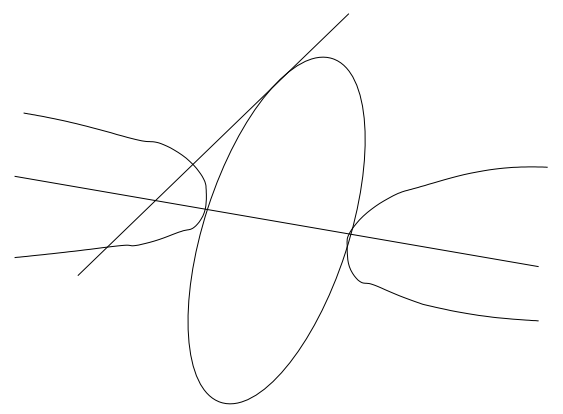

FiguRE 31. Fourth case

Using braid monodromy techniques and the van Kampen theorem, we get the following presentation for the fundamental group of the complement:

Generators: $\left\{x_{1}, x_{2}, x_{3}, x_{4}, x_{5}, x_{6}\right\}$.

Relations:

(1) $x_{6} x_{5} x_{4} x_{3} x_{2} x_{1}=e$ (projective relation)

(2) $x_{4}=x_{5}$

(3) $x_{1}=x_{4} x_{3} x_{4}^{-1}$

(4) $x_{4} x_{3} x_{2}=x_{2} x_{4} x_{3}$

(5) $x_{4} x_{3} x_{2} x_{4} x_{3}=x_{3} x_{2} x_{4} x_{3} x_{4}$

(6) $\left(x_{4} x_{6}\right)^{2}=\left(x_{6} x_{4}\right)^{2}$

(7) $x_{4} x_{3} x_{4}^{-1} x_{4} x_{2} x_{4} x_{3} x_{4}^{-1} x_{4}=x_{4} x_{2} x_{4} x_{3} x_{4}^{-1} x_{4} x_{4} x_{3} x_{4}^{-1}$

(8) $x_{2} x_{4} x_{3} x_{4}^{-1} x_{4}=x_{4} x_{3} x_{4}^{-1} x_{4} x_{2}$

(9) $x_{4} x_{3} x_{4}^{-1}=x_{2}^{-1} x_{3}^{-1} x_{4}^{-1} x_{6} x_{4}^{-1} x_{6}^{-1} x_{4} x_{3} x_{4}^{-1} x_{6} x_{4} x_{6}^{-1} x_{4} x_{3} x_{2}$

(10) $x_{2}^{-1} x_{3}^{-1} x_{4}^{-1} x_{6} x_{4} x_{6}^{-1} x_{4} x_{3} x_{2}=x_{4} x_{3} x_{4}^{-1} x_{4} x_{4} x_{3}^{-1} x_{4}^{-1}$

(11) $x_{4} x_{1} x_{4}^{-1} x_{6}=x_{6} x_{4} x_{1} x_{4}^{-1}$

(12) $x_{4} x_{2} x_{4}^{-1} x_{6}=x_{6} x_{4} x_{2} x_{4}^{-1}$

(13) $x_{5} x_{3} x_{5}^{-1} x_{6}=x_{6} x_{5} x_{3} x_{5}^{-1}$

Using Relations (2) and (3), we can cancel generators $x_{1}$ and $x_{5}$, and replace them by $x_{4} x_{3} x_{4}^{-1}$ and $x_{4}$ respectively. By a trivial simplification, Relation (8) is equal to Relation (4), so it is redundant. So we get the following simplified presentation:

Generators: $\left\{x_{2}, x_{3}, x_{4}, x_{6}\right\}$.

Relations: 
(1) $x_{6} x_{4}^{2} x_{3} x_{2} x_{4} x_{3} x_{4}^{-1}=e$

(2) $x_{4} x_{3} x_{2}=x_{2} x_{4} x_{3}$

(3) $x_{4} x_{3} x_{2} x_{4} x_{3}=x_{3} x_{2} x_{4} x_{3} x_{4}$

(4) $\left(x_{4} x_{6}\right)^{2}=\left(x_{6} x_{4}\right)^{2}$

(5) $x_{3} x_{2} x_{4} x_{3}=x_{2} x_{4} x_{3} x_{4} x_{3} x_{4}^{-1}$

(6) $x_{4} x_{3} x_{4}^{-1}=x_{2}^{-1} x_{3}^{-1} x_{4}^{-1} x_{6} x_{4}^{-1} x_{6}^{-1} x_{4} x_{3} x_{4}^{-1} x_{6} x_{4} x_{6}^{-1} x_{4} x_{3} x_{2}$

(7) $x_{2}^{-1} x_{3}^{-1} x_{4}^{-1} x_{6} x_{4} x_{6}^{-1} x_{4} x_{3} x_{2}=x_{4} x_{3} x_{4} x_{3}^{-1} x_{4}^{-1}$

(8) $x_{4}^{2} x_{3} x_{4}^{-2} x_{6}=x_{6} x_{4}^{2} x_{3} x_{4}^{-2}$

(9) $x_{4} x_{2} x_{4}^{-1} x_{6}=x_{6} x_{4} x_{2} x_{4}^{-1}$

(10) $x_{4} x_{3} x_{4}^{-1} x_{6}=x_{6} x_{4} x_{3} x_{4}^{-1}$

From Relation (5) we have $x_{3} x_{2} x_{4} x_{3} x_{4}=x_{2} x_{4} x_{3} x_{4} x_{3}$ which is equal to Relation (3) by Relation (2). Hence, Relation (5) is redundant.

Also, Relation (8) follows immediately from Relation (10), and hence it is redudant too.

Since the right side of Relation (1) is $e$, we can move $x_{4} x_{3} x_{4}^{-1}$ to the left, in order to get: $x_{4} x_{3} x_{4}^{-1} x_{6} x_{4}^{2} x_{3} x_{2}=e$. By Relation (10) and a cancellation, we have $x_{6}\left(x_{4} x_{3}\right)^{2} x_{2}=e$.

Hence, we get the following presentation:

Generators: $\left\{x_{2}, x_{3}, x_{4}, x_{6}\right\}$.

Relations:

(1) $x_{6}\left(x_{4} x_{3}\right)^{2} x_{2}=e$

(2) $x_{4} x_{3} x_{2}=x_{2} x_{4} x_{3}$

(3) $x_{4} x_{3} x_{2} x_{4} x_{3}=x_{3} x_{2} x_{4} x_{3} x_{4}$

(4) $\left(x_{4} x_{6}\right)^{2}=\left(x_{6} x_{4}\right)^{2}$

(5) $x_{4} x_{3} x_{4}^{-1}=x_{2}^{-1} x_{3}^{-1} x_{4}^{-1} x_{6} x_{4}^{-1} x_{6}^{-1} x_{4} x_{3} x_{4}^{-1} x_{6} x_{4} x_{6}^{-1} x_{4} x_{3} x_{2}$

(6) $x_{2}^{-1} x_{3}^{-1} x_{4}^{-1} x_{6} x_{4} x_{6}^{-1} x_{4} x_{3} x_{2}=x_{4} x_{3} x_{4} x_{3}^{-1} x_{4}^{-1}$

(7) $x_{4} x_{2} x_{4}^{-1} x_{6}=x_{6} x_{4} x_{2} x_{4}^{-1}$

(8) $x_{4} x_{3} x_{4}^{-1} x_{6}=x_{6} x_{4} x_{3} x_{4}^{-1}$

By the first relation $x_{2}=\left(x_{4} x_{3}\right)^{-2} x_{6}^{-1}$, so we can replace $x_{2}$ by $\left(x_{4} x_{3}\right)^{-2} x_{6}$ in any place it appears. We start with Relation (7). We have: $x_{4}\left(x_{4} x_{3}\right)^{-2} x_{6}^{-1} x_{4}^{-1} x_{6}=x_{6} x_{4}\left(x_{4} x_{3}\right)^{-2} x_{6}^{-1} x_{4}^{-1}$. By some simplifications, we have: $x_{6} x_{4} x_{6} x_{4} x_{3} x_{4} x_{3}=x_{4} x_{6} x_{4} x_{3} x_{4} x_{3} x_{4}^{-1} x_{6} x_{4}$ By Relations (4) and (8), we have: $x_{6} x_{3}=x_{3} x_{6}$.

Relation (2) gets the following form: $x_{4} x_{3}\left(x_{4} x_{3}\right)^{-2} x_{6}^{-1}=\left(x_{4} x_{3}\right)^{-2} x_{6}^{-1} x_{4} x_{3}$. By some simplifications, we have: $x_{4} x_{3} x_{6}=x_{6} x_{4} x_{3}$. By the relation $x_{6} x_{3}=x_{3} x_{6}$, we finally have that: $x_{4} x_{6}=x_{6} x_{4}$. Therefore, Relation (4) is now redundant. Moreover, Relation (8) can be reduced to $x_{3} x_{6}=x_{6} x_{3}$, which is known, and hence Relation (8) is redundant too.

Since $x_{3}$ and $x_{4}$ commute with $x_{6}$, Relations (5) and (6) get the following forms respectively: $x_{4} x_{3} x_{4}^{-1}=\left(x_{6}\left(x_{4} x_{3}\right)^{2}\right) x_{3}^{-1} x_{4}^{-1} x_{3} x_{4} x_{3}\left(\left(x_{4} x_{3}\right)^{-2} x_{6}^{-1}\right)$ and $\left(x_{6}\left(x_{4} x_{3}\right)^{2}\right) x_{3}^{-1} x_{4} x_{3}\left(\left(x_{4} x_{3}\right)^{-2} x_{6}^{-1}\right)=x_{4} x_{3} x_{4} x_{3}^{-1} x_{4}^{-1}$. If we continue 
the simplifications, we get that both relations become trivial, and hence they are redundant.

Finally, we simplify Relation (3). By Relation (1), it gets the following form: $x_{4} x_{3}\left(\left(x_{4} x_{3}\right)^{-2} x_{6}^{-1}\right) x_{4} x_{3}=x_{3}\left(\left(x_{4} x_{3}\right)^{-2} x_{6}^{-1}\right) x_{4} x_{3} x_{4}$. By some simplifications, we get that this relation becomes trivial, and hence it is redundant too.

Therefore, we get the following simplified presentation:

Generators: $\left\{x_{3}, x_{4}, x_{6}\right\}$.

Relations:

(1) $x_{3} x_{6}=x_{6} x_{3}$

(2) $x_{4} x_{6}=x_{6} x_{4}$

This group is obviously the group:

$$
\mathbb{Z} \oplus \mathbb{F}_{2}
$$

as needed.

\section{The Fundamental Groups ARE BIG}

A group is called big if it contains a subgroup which is free (generated by two or more generators). In this section we show that the fundamental groups which appear in this paper are big.

We start with a simple observation. If $G$ is a group which has a big quotient, then $G$ is big itself: Let $N$ be a normal subgroup of $G$ such that $G / N$ is big. Let $a N, b N$ be the two generators of the free subgroup of $G / N$, then the group generated by $a, b$ in $G$ is big. Otherwise, there is a relation $w(a, b)=1$ in $G$, and therefore we have a corresponding relation $w(a N, b N)=N$, which is a contradiction to the freeness of the subgroup $\langle a N, b N\rangle \leq G / N$.

Using the observation, we show the following result:

Proposition 6.1. The group

$$
G \cong\left\langle a, b \mid(a b)^{2}=(b a)^{2}=e\right\rangle
$$

is big.

Proof. First, we change the presentation: let us take new generators $x=a b, y=b$, then the relation $(a b)^{2}=(b a)^{2}=e$ becomes: $x^{2}=$ $\left(y x y^{-1}\right)^{2}=e$, which is equal to: $x^{2}=y x^{2} y^{-1}=e$. Hence, we have:

$$
G \cong\left\langle x, y \mid x^{2}=y x^{2} y^{-1}=e\right\rangle
$$

Now, the quotient of $G$ by the subgroup generated by $y^{3}$ is

$$
G /\left\langle\left\langle y^{3}\right\rangle\right\rangle \cong \mathbb{Z} / 2 * \mathbb{Z} / 3
$$

where $*$ is the free product. 
Since $\mathbb{Z} / 2 * \mathbb{Z} / 3$ is known to be big, by the observation we have that $G$ is big as needed.

Now we can prove Corollary 1.4. Let $A$ be a conic-line arrangement with two tangent conics and up to two additional lines. Since the two tangent conics is a sub-arrangement of $A$, it is known that the fundamental group of the two tangent conics is a quotient of the fundamental group of $A$ (by sending the generators which correspond to the additional lines to $e$ ). Since, we have shown in the previous proposition that the fundamental group of the two tangent conics is big, then we have that the fundamental group of $A$ is big too (by using again the observation). Hence, we are done.

\section{ConjeCtures CONCERning the CASE of ARRAngements WITH ONLY SIMPLE TANGENCY POINTS}

Motivated by the presentations we achieved in the cases of two tangent conics, with up to two tangent lines with only simple tangnecy points, we want to propose some conjectures about the simplified presentation of the fundamental group of two tangent conics, with an arbitrary number of tangented lines, which are tangent in simple tangency points.

Conjecture 7.1. Let $S$ be a conic-line arrangement with two tangent conics, and $n$ lines which are tangent to these conics. Assume that the tangency points of $S$ are simple. Let $C_{1}$ be the conic with the maximal number $m$ of lines which are tangent to it. Then the following holds in the simplified presentation of $\pi_{1}\left(\mathbb{C P}^{2}-S\right)$ :

(1) We have $m$ relations of the type

$$
\left(a x_{i}\right)^{2}=\left(x_{i} a\right)^{2},
$$

where $a$ is the generator corresponding to the conic $C_{1}$ and $x_{i}$ are some generators of the group.

(2) Let $y$ and $z$ be two generators of $\pi_{1}\left(\mathbb{C P}^{2}-S\right)$ which are different from a. We have

$$
y a z a^{-1}=a z a^{-1} y
$$

Remark 7.2. In some of the computed presentations, we do not have all the relations of the type mentioned in the second part of the conjecture. This happens since some of these relations are simplified to commutative relations between generators by other relations.

For a specific case, we should get a decomposition: 
Conjecture 7.3. Let $S$ be a conic-line arrangement with two tangent conics and $n$ lines, where $n-1$ lines are tangent to one conic, and one line $L$ is tangent to the other one. Assume that all the tangency points are simple. Let $x$ be the generator that corresponds to L. Then:

$$
\pi_{1}\left(\mathbb{C P}^{2}-S\right)=\langle x\rangle \oplus \pi_{1}\left(\mathbb{C P}^{2}-(S-L)\right) .
$$

\section{APPENDIX}

In the appendix, we list all the possibilities for two tangency points and two additional lines, and the corresponding fundmanetal groups of the complement (we assume that the line at infinity is transversal to our curve, i.e. there are no singularities on the line at infinity).

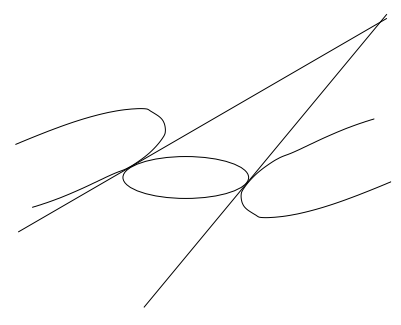

$$
\pi_{1}\left(\mathbb{C P}^{2}-S\right) \cong\left\langle x, y, z \mid(z y x)^{2}=(y x z)^{2}=(x z y)^{2}\right\rangle
$$
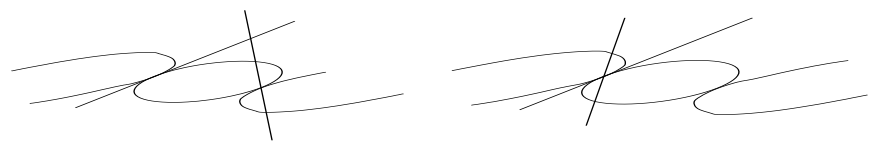

(2)
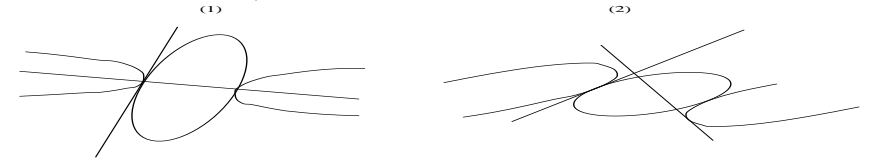

(3)
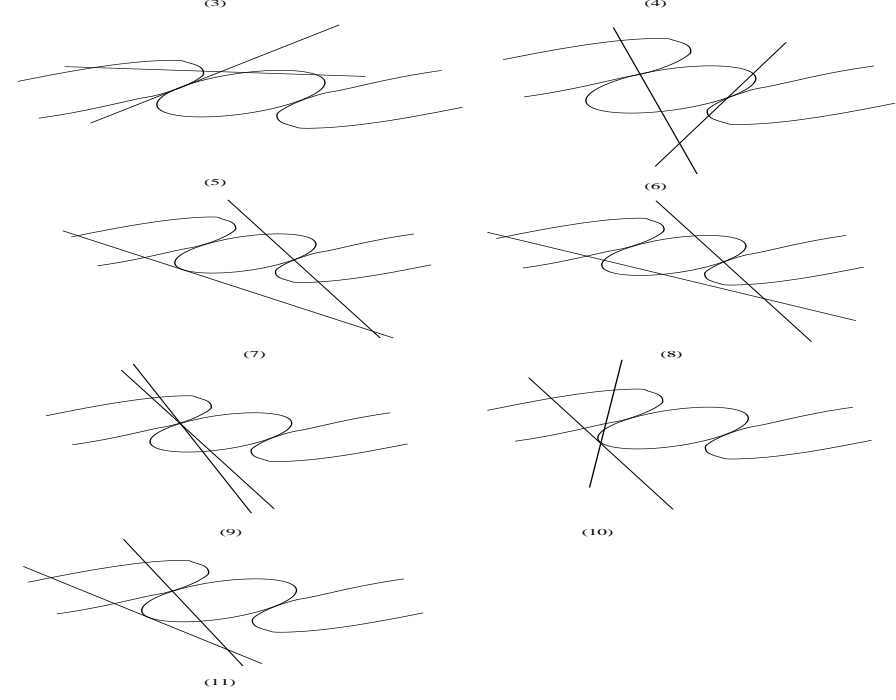


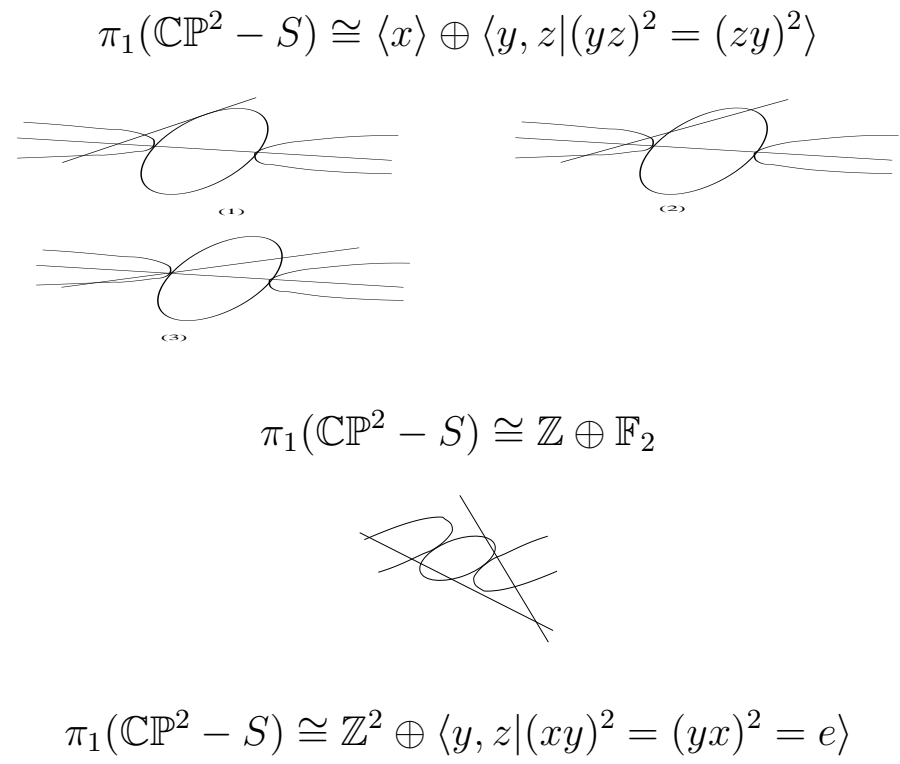

ACKNOWLEDGMENTS

We wish to thank Alex Degtyarev for fruitful discussions.

The first and second authors wish to thank the Institute of Mathematics, Erlangen (Germany), Institut Fourier, Grenoble (France), Hershel Farkas, Ron Livne and Einstein Institute of Mathematics for hosting their stays.

\section{REFERENCES}

[1] Amram, M. and Teicher, M., Braid monodromy of special curves, J. Knot Theory \& Ramifications 10(2), 171-212 (2001).

[2] Degtyarev, A. I., Quintics in $\mathbb{C P}^{2}$ with non-abelian fundamental group, Algebra i Analiz 11(5), 130-151 (1999); Translation in: St. Petersburg Math. J. 11(5), 809-826 (2000).

[3] Garber, D., On the connection between affine and projective fundamental groups of line arrangements and curves, Singularités Franco-Japonaises (J.P. Brasselet and T.Suwa, eds.), Séminaires \& Congrès 10, 61-70 (2005).

[4] Garber, D. and Teicher, M., The fundamental group's structure of the complement of some configurations of real line arrangements, Complex Analysis and Algebraic Geometry, edited by T. Peternell and F.-O. Schreyer, de Gruyter, 173-223 (2000).

[5] Hirzebruch, F., Some examples of algebraic surfaces, Contemp. Math. 9, 55-71 (1982).

[6] Moishezon, B., Stable branch curves and braid monodromies, Lect. Notes in Math. 862, 107-192 (1981).

[7] Moishezon, B. and Teicher, M., Braid group techniques in complex geometry I, Line arrangements in $\mathbb{C P}^{2}$, Contemp. Math. 78, 425-555 (1988). 
[8] Moishezon, B. and Teicher, M., Braid group techniques in complex geometry II, From arrangements of lines and conics to cuspidal curves, Algebraic Geometry, Lect. Notes in Math. 1479, 131-180 (1990).

[9] Moishezon, B. and Teicher, M., Braid group technique in complex geometry III, Projective degeneration of $V_{3}$, Contemp. Math. 162, 313-332 (1994).

[10] Moishezon, B. and Teicher, M., Braid group technique in complex geometry $I V$, Braid monodromy of the branch curve $S_{3}$ of $V_{3} \rightarrow \mathbb{C P}^{2}$ and application to $\pi_{1}\left(\mathbb{C P}^{2}-S_{3}, *\right)$, Contemp. Math. 162, 332-358 (1994).

[11] van Kampen, E. R., On the fundamental group of an algebraic curve, Amer. J. Math. 55, 255-260 (1933).

Meirav Amram, Einstein Institute of Mathematics, The Hebrew UniVERsity, Givat RAm, 91904 JERUSALEM, ISRAEL

E-mail address: ameirav@math.huji.ac.il

David Garber, Einstein Institute of Mathematics, The Hebrew UniVersity, Givat Ram, 91904 Jerusalem, IsRael, And Department of Applied Mathematics, Holon Academic Institute of Technology, 52 Golomb St., PO Box 305, 58102 Holon, IsRAEL

E-mail address: garber@math.huji.ac.il,garber@hait.ac.il

Mina Teicher, Department of Mathematics, Bar-Ilan University, 52900 RAMAT GAN, ISRAEL

E-mail address: teicher@macs.biu.ac.il 
ta be

$\dot{p} \in \dot{\infty}$

$\dot{x}=$ 
of $x$

书书 $\infty$ 